\title{
ACYCLIC MODELS AND FIBRE SPACES
}

\author{
BY \\ V. K. A. M. GUGENHEIM(1) AND J. C. MOORE $\left({ }^{2}\right)$
}

Introduction. Most of the recent rapid development of the homology or cohomology theory of fibre spaces is based on the use of spectral sequences, as introduced by J. Leray [1 ]. In order to use spectral sequences successfully, it is almost always necessary to have a good deal of information about the term usually called for cohomology $E_{2}$ or for homology $E^{2}$ of the spectral sequence. In his original work, Leray proved that the term $E_{2}$ in the spectral sequence for the Cech cohomology of a fibre space is naturally isomorphic to the cohomology of the base space with local coefficients in the cohomology of the fibre. The appropriate analogues of this result for cubical singular homology, and cohomology were proved by J.-P. Serre [3], and are fundamental in applications of homology theory to homotopy theory.

The main object of this paper is to calculate the term $E^{2}$ in the singular homology spectral sequence of a fibre space by the use of the theory of acyclic. models of Eilenberg and MacLane [4]. Notice that we said the singular homology spectral sequence of a fibre space, and did not specify whether we meant simplicial or cubical singular theory. One of our results asserts essentially that there is a singular homology spectral sequence of a fibre space, and that it may be obtained by using either simplicial or cubical singular theory. Further, we show that in cubical singular homology theory a filtration somewhat different from the one used by Serre may be used to obtain the spectral sequence. This filtration is symmetric in all coordinates, and consequently much more convenient to use when dealing with fibre spaces wherein a multiplication is defined in the base space and total space, and such that projection map is a homomorphism. This fact will be exploited elsewhere.

In the course of our work, it has been necessary to make a fairly extensive axiomatic investigation of the notion of a "singular theory," and of the notion of "local coefficients." One outcome of this work is a proof of the fact that cubical singular homology and simplicial singular homology coincide even with local coefficients. For ordinary coefficients this was proved by Eilenberg and MacLane [4].

Since much of our work is quite abstract, the general theory will be interspersed with examples from cubical singular theory.

Technically this paper is divided into chapters, and each chapter into sections. The notation II.3.4 refers to the 4 th numbered statement in the

Received by the editors October 20, 1955 and, in revised form, June 8, 1956.

(1) Commonwealth Fund Fellow, 1952-1954.

(2) National Science Foundation Fellow, 1953-1955. 
third section of Chapter II; within Chapter II, this statement will be referred to as 3.4.

\section{General abstract notions}

1. Categories and functors. The language of this paper is that of "categories and functors," cf. [6] and [7]. We recall briefly the principal notions.

A category is a collection $Q$ consisting of "objects" and "maps"; the general nature is indicated sufficiently by such examples as "topological spaces and continuous maps," and "groups and homomorphisms."

If $f: A \rightarrow B$ is a map, we call $A$ the domain, and $B$ the range of $f$. If $f: A \rightarrow B$ and $g: B \rightarrow C$ are maps, $g f: A \rightarrow C$ is the "composition." With any object $A$ is associated the "identity" $1(A): A \rightarrow A$.

The symbol 1 will be used ambiguously to denote any identity the object of which is clear from the context.

Let $Q$ and $B$ be categories. A function $T$ which assigns to each object $A$ of $Q$ an object $T(A)$ of $B$, and to each map $f: A \rightarrow B$ of $A$ a map $T(f): T(A)$ $\rightarrow T(B)$ of $B$ will be called a covariant functor $T: Q \rightarrow B$ if

(i) $T(g f)=T(g) T(f)$ whenever $g f$ is defined,

(ii) $T(1(A))=1(T(A))$ for any object $A$ of $\propto$.

This is the only kind of functor used in this paper to any great extent. The functor $a \rightarrow Q$ which assigns to any object or map that object or map itself will again, ambiguously, be denoted by 1 .

If $Q^{\prime}$ is a sub-category of $Q$, and $T: Q \rightarrow B$ a functor, $T \mid Q^{\prime}$ denotes the restriction of $T$ to $Q^{\prime}$.

Let $S, T: Q \rightarrow B$ be covariant functors. A function $\Gamma$ which assigns to each object $A$ of $Q$ a map $\Gamma(A): S(A) \rightarrow T(A)$ of $B$ will be called a "natural transformation of functors $\Gamma: S \rightarrow T$ if for every map $f: A \rightarrow B$ the "condition of naturality" $\Gamma(B) S(f)=T(f) \Gamma(A)$ is satisfied. For most natural transformations of functors the verification of naturality is easy. Such a verification will usually be omitted in this paper; sometimes it is quite tedious. In one case in Chapter II, the verification of naturality is difficult, and constitutes the crux of the whole argument. Here the verification will be done in detail.

The natural transformation $T \rightarrow T$ which assigns $1(T(A))$ to the object $A$ is called the identity transformation, and will be denoted by 1 .

If $S, T: Q \rightarrow B$ are functors, and $Q^{\prime}$ a sub-category of $Q$, the restriction of $\Gamma$ to $S \mid \mathfrak{Q}^{\prime}$ will be denoted by $\Gamma \mid \mathfrak{Q}^{\prime}$. If $\beta$ is a category in which a notion of inclusion $\subset$ is defined, we shall write $S \subset T$ if $S(A) \subset T(A)$ for every object $A$ of $Q$.

2. The derived category. Let $a$ be a category. By $D \propto$ we shall denote the category defined as follows:

(i) the objects of $D Q$ are the maps of $Q$, and

(ii) the maps of $D Q$ are quadruples $(u, v ; f, g)$ where $u, v, f, g$ are maps of a such that $g u=v f$.

The category $D Q$ is known as the derived category of $Q$. A map, $(u, v: f, g)$, 
in this category is a commutative diagram

$$
\begin{gathered}
A \stackrel{u}{\rightarrow} B \\
f \downarrow \stackrel{v}{ } \stackrel{\downarrow}{\rightarrow} \\
A^{\prime} \stackrel{\rightarrow}{\rightarrow} B^{\prime}
\end{gathered}
$$

in $Q$. The domain of $(u, v ; f, g)$ is $f$, and the range is $g$.

If $(u, v ; f, g),\left(u^{\prime}, v^{\prime} ; g, h\right)$ are maps, composition is defined by $\left(u^{\prime}, v^{\prime} ; g, h\right)$ $\cdot(u, v ; f, g)=\left(u^{\prime} u, v^{\prime} v ; f, h\right)$, and if $f: A \rightarrow B$ is an object of $D Q$, we define $1(f)=(1(A), 1(B) ; f, f)$.

The category $D Q$ and various sub-categories will play an important role later in this paper. Actually, we will sometimes consider even more complicated categories obtained from $a$, such as, for example, the derived category of the derived category of $Q$, denoted by DDQ.

Sometimes we want to take direct sums of modules indexed on something rather large. We will assume that we are using some sort of set theory where this makes sense. For example, if we use the system of von NeumannBernays-Gödel which distinguishes between "sets" and "classes" it is sufficient to assume

(i) the objects of the category $\mathfrak{T}$ (I.1.1) form a set, and

(ii) if $A, B$ are objects of the category $Q$, the class of maps $f: A \rightarrow B$ is a set.

Condition (ii) is satisfied in all categories where the objects are sets of elements. Condition (i) is satisfied in the singular theories of Chapter III, but not for all the examples of Chapter II. This, however, causes no difficulties.

\section{ACyClic MOdELS AND LOCAL COEFFICIENTS}

1. Acyclic models. If $Q$ is a category and $\mathfrak{T}$ a sub-category of $Q$, we shall denote by $[\mathfrak{T}, Q]$ the set of all mappings of $Q$ with domain an object of $\mathfrak{T}$, and by $[\mathfrak{M}, A]$ the subset of $[\mathfrak{T}, Q]$ whose range is a given object $A$ of $a$.

1.1. Definitions. The pair $(Q, \mathfrak{T})$ ) will be called a category with models if $Q$ is a category, and $\mathfrak{T}$ is a sub-category of $Q$ called the model sub-category. Objects of $\mathfrak{T C}$ are called models or model objects.

The quadruple $(\alpha, \mathfrak{T}, \alpha, \beta)$ will be called a category with models and degeneracies if $(\alpha, \mathfrak{T})$ ) is a category with models, and $\alpha$ and $\beta$ are functions mapping $[\mathfrak{T}, Q]$ into itself such that

(0) $\alpha(1(M))=\beta(1(M))=1(M)$ if $M$ is a model,

(1) $\beta(u) \alpha(u)=u$, where $\alpha(u)$ is a map in $\Re$,

(2) $\alpha(\beta(u))=\beta(\alpha(u))=1(M)$ where $M$ is the domain of $\beta(u)$ and the range of $\alpha(u)$,

(3) $\beta(f u)=\beta(f \beta(u))$ where $f$ is a mapping of $A$ such that domain $f=$ range $u$, and

(4) $\alpha(f u)=\alpha(f \beta(u)) \alpha(u)$ (where $f$ is as in 3 ). 
Notice that (3) implies that $\beta(\beta(u))=\beta(u)$, and that (1) and (2) imply that $\alpha(\alpha(u))=\alpha(u)$.

The axioms concerning $\alpha$ and $\beta$ in the preceding definition say that if $u: M \rightarrow A$ is a map in $Q$ whose domain is a model object, then $u$ can be factored into $\beta(u) \alpha(u)$ where $\alpha(u): M \rightarrow M^{\prime}$ is a map in $\mathscr{T}$, and $\beta(u): M^{\prime} \rightarrow A$. Further suppose that $f: A \rightarrow B$ is a map in $Q$, we then have the diagram

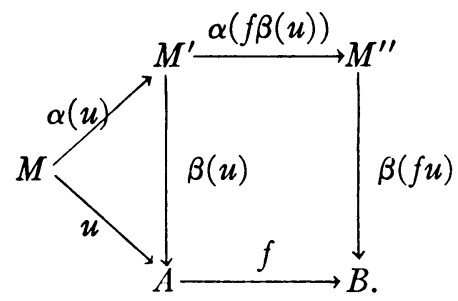

This diagram is commutative, and axioms (3) and (4) give the relationship between the factorization of $u$ and the factorization of $f u$.

The functions $\alpha$ and $\beta$ are known as a system of degeneracies on the category with models $(Q, \mathfrak{T})$. Any such category may be provided with a system of degeneracies in a trivial fashion by setting $\alpha(u)=1(M)$ and $\beta(u)=u$ for any map $u: M \rightarrow A$ with domain a model.

1.2. Example. Suppose for a moment that the category $a$ with which we are dealing is the category of topological spaces and continuous maps. Let $I^{0}$ be a point, and for $n>0$, let $I^{n}$ be the standard $n$-cube, i.e. $I^{n}$ consists of $n$ tuples of real numbers $\left(t_{1}, \cdots, t_{n}\right)$ such that $0 \leq t_{i} \leq 1$ for $i=1, \cdots, n$. The model objects are the cubes $\left\{I^{n}\right\}$, and $\mathscr{N}$ is the category of continuous maps with domain and range a cube.

If $u: I^{n} \rightarrow A$ is a map, we must factor $u$ into $\beta(u) \alpha(u)$. Suppose that $u\left(t_{1}, \cdots, t_{n}\right)=u\left(t_{1}, \cdots, t_{p}, 0, \cdots, 0\right)$ and that $p$ is the smallest integer for which this is true. Define $\alpha(u): I^{n} \rightarrow I^{p}$ by $\alpha(u)\left(t_{1}, \cdots, t_{n}\right)=\left(t_{1}, \cdots, t_{p}\right)$, and $\beta(u): I^{p} \rightarrow A$ by $\beta(u)\left(t_{1}, \cdots, t_{p}\right)=u\left(t_{1}, \cdots, t_{p}, 0, \cdots, 0\right)$. One verifies easily that with these definitions $\alpha$ and $\beta$ are a system of degeneracies.

Assumption. For the rest of this section $(Q, \mathfrak{M})$ is a fixed category with models; it will usually be denoted by $a$, object will mean object of $a$, and mapping will mean mapping of $a$. Moreover, system of degeneracies will mean such a system (1.1) on the category $(\alpha, \mathfrak{T})$.

1.3. Definitions. If $\alpha, \beta$ is a system of degeneracies on $\alpha$, then $u \in[\mathfrak{T}, Q]$ is degenerate if $u \neq \beta(u)$, and is nondegenerate if $u=\beta(u)$. For an object $A$, let $S(A)$ denote the set of nondegenerate maps in $[\mathfrak{T}, A]$. The system of degeneracies being used will always be clear from the context.

Assumptions and notations. For the rest of this paper we will work over a fixed commutative ring with unit, denoted when necessary by $\Lambda$. All notions such as homomorphism, module, or tensor product will be relative to $\wedge$. The category of $\Lambda$-modules and $\Lambda$-homomorphisms will be denoted by $\mathcal{G}_{\Lambda}^{\prime}$. 
However, since $\Lambda$ is fixed this will usually be abbreviated to $\mathcal{G}^{\prime}$. Further the category of graded $\Lambda$-modules and graded $\Lambda$-homomorphisms will be denoted by $G_{\Lambda}$, or $g$. An object in this category is a $\Lambda$-module $G$ which is the direct sum of submodules indexed on the non-negative integers, i.e. $G$ $=\sum_{n \geq 0} G_{n}$, and a homomorphism $\phi: G \rightarrow G^{\prime}$ is a homomorphism of modules such that $\phi\left(G_{n}\right) \subset G_{n}^{\prime}$ for all $n$. We will denote $\phi \mid G_{n}$ by $\phi_{n}$. The function which takes $G$ into $G_{n}$ for $G$ an object in $\mathcal{G}$, and $\phi$ into $\phi_{n}$ for $\phi$ a map in $\mathcal{G}$ is a covariant functor taking $\mathcal{G}$ into $\mathcal{G}^{\prime}$. There is also another covariant functor taking $\mathcal{G}$ into $\mathcal{G}^{\prime}$ which is sometimes convenient to use, namely the functor which forgets about gradation.

The category $\mathcal{G}^{\prime}$ is embedded in the category $\mathcal{G}$ in a natural way by mapping a module $G$ into the graded module $G^{\prime}$ such that $G_{0}^{\prime}=G$, and $G_{n}^{\prime}=0$ for $n>0$.

In the category $G$ the tensor product of two modules $G$ and $G^{\prime}$ is the module $G \otimes G^{\prime}$ such that $\left(G \otimes G^{\prime}\right)_{n}=\sum_{r+s=n} G_{r} \otimes G_{s}^{\prime}$. Similarly if $\phi$ and $\phi^{\prime}$ are homomorphism in $\mathcal{G}$, then $\left(\phi \otimes \phi^{\prime}\right)_{n}=\sum_{r+\varepsilon=n} \phi_{r} \otimes \phi_{s}^{\prime}$.

Finally in the category 9 we have the notion of homomorphism of degree $r$, where $r$ is an integer. A homomorphism of degree $r, \phi: G \rightarrow G^{\prime}$ is a homomorphism of modules such that $\phi\left(G_{n}\right) \subset G_{n+r}{ }^{\prime}$. Actually we should say that this is the case so long as $n+r$ is greater than or equal to zero. Instead we adopt the convention that an object in $\mathcal{Q}$ has a submodule $G_{n}$ for each integer

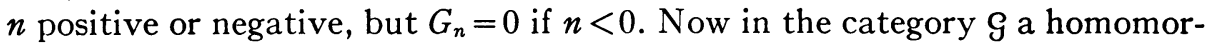
phism is the same thing as a homomorphism of degree 0 .

1.4. Definitions. If $K: Q \rightarrow \mathcal{G}$ is a covariant functor, and $u: M \rightarrow A$ is an element of $[\Re, A]$, we shall denote by $K(M, u)$ the set of pairs $(k, u)$ such that $k \in K(M)$. Let $i(u): K(M) \rightarrow K(M, u)$ be defined by $i(u) k=(k, u)$, and let $j(u)$ be the inverse of $i(u)$. The set $K(M, u)$ is to be considered as a module so that $i(u)$ is an isomorphism.

Let $\alpha, \beta$ be a system of degeneracies, and define a new functor $\hat{K}: a \rightarrow \mathcal{G}$ by setting $\hat{K}(A)=\sum_{u \in S(A)} K(M, u)$ for any object $A$, and $K(f) \mid K(M, u)$ $=i(\beta(f u)) K(\alpha(f u)) j(u)$ for any map $f: A \rightarrow B$. Evidently $K(f)(K(M, u))$ $\subset K\left(M^{\prime}, \beta(f u)\right)$ where $M^{\prime}$ is the domain of $\beta(f u)$, and $\beta(f u) \in S(B)$ as required.

Next we define a natural transformation of functors $\Gamma_{K}: \hat{K} \rightarrow K$ by setting $\Gamma_{K}(A) \mid K(M, u)=K(u) j(u)$ for $A$ an object and $u \in S(A)$. The functor $K$ is representable relative to the system of degeneracies $\alpha, \beta$ if there is a natural transformation of functors $\chi_{K}: K \rightarrow \hat{K}$ such that $\Gamma_{K} \chi_{K}: K \rightarrow K$ is the identity. When no confusion will result, $\Gamma_{K}$ is abbreviated by $\Gamma$, and $\chi_{K}$ by $\chi$.

Notice that if $A$ is an object, then $\hat{K}(A)$ does not depend on the system of degeneracies being used, but that if $f: A \rightarrow B$ is a map then $\hat{K}(f)$ does depend on the system of degeneracies. Further, notice that representability is a notion which depends on the system of degeneracies. The question of what system of degeneracies is convenient to use when dealing with a given functor $K$ is closely tied up with the structure of the functor $K$, as we shall see later. 
1.5. Notation And conventions. Let $d g$ denote the category of differential graded modules and admissable homomorphism; in other words an object of $d \mathcal{G}$ is a pair $(G, d)$ such that $G$ is an object of $\mathcal{G}$ and $d$ is an endomorphism of degree -1 of $G$ such that $d d=0$. A map $f$ is a map of graded modules which has the additional property that $f d=d f$. Hereafter an object $(G, d)$ in $d \mathcal{G}$ will be denoted merely by $G$. There is a covariant functor taking the category $d \mathcal{G}$

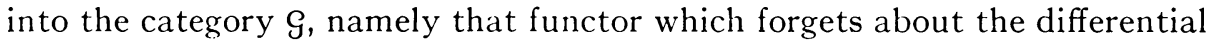
operator. If $K: a \rightarrow d \mathcal{G}$ is a covariant functor, we will use the same notation for the associated covariant functor taking $Q$ into $\mathcal{G}$, and if $L: Q \rightarrow d \mathcal{G}$ is another such functor, we say that $T: K \rightarrow L$ is a natural transformation of functors of degree $r \neq 0$ if it is a natural transformation of the associated functors mapping into $\mathcal{G}$, and $T: K_{n} \rightarrow L_{n+r}$. In the category $d \mathcal{G}$ homology is defined as usual, and we have that $H(G)=\sum_{n \geq 0} H_{n}(G)$. Note that $H$ is a covariant functor mapping $d \mathcal{G}$ into $g$. The natural transformation of functors $G_{0} \rightarrow H_{0} G$ will always be denoted by $\epsilon$.

1.6. Definition. If $K: Q \rightarrow d \subseteq$ is a covariant functor, and $\alpha, \beta$ is a system of degeneracies on $Q$, we say that $K$ is representable if the associated functor $K: Q \rightarrow g$ is representable (1.4).

1.7. Example. Again suppose $Q$ is the category of topological spaces and continuous maps with models as in 1.2. For any object $A$, define $Q_{n}(A)$ to be the free module generated by the maps $u: I^{n} \rightarrow A$. Such a map is called a singular $n$-cube of $A$. Let $D_{n}(A)$ be the submodule of $Q_{n}(A)$ generated by the degenerate $n$-cubes of $A$, i.e. those such that $u \neq \beta(u)$, and let $C_{n}(A)$ $=Q_{n}(A) / D_{n}(A)$. Further, let $C(A)=\sum_{n \geq 0} C_{n}(A)$. If $u: I^{n} \rightarrow A$ is a singular $n$-cube, define $\lambda_{i}^{i} u: I^{n-1} \rightarrow A$ by $\lambda_{i}^{i} u\left(t_{1}, \cdots, t_{n-1}\right)=u\left(t_{1}, \cdots, t_{i-1}, \quad \epsilon\right.$, $\left.t_{i+1}, \cdots, t_{n-1}\right)$ where $\epsilon=0$ or 1 , and $i=1, \cdots, n$. Let $d: Q_{n} \rightarrow Q_{n-1}$ be defined by $d u=\sum_{i=0}^{n}(-1)^{i}\left(\lambda_{i}^{0} u-\lambda_{i}^{1} u\right)$. Observe that $d$ induces an endomorphism still called $d$ of $C(A)$, and $d^{2}=0$.

If $f: A \rightarrow B$ is a map, define $C(f): C(A) \rightarrow C(B)$ by $C(f) u=f u$ for $u$ a singular $n$-cube of $A$. One verifies easily that $C: Q \rightarrow d \mathcal{G}$ is a covariant functor. Further $C_{n}(A)$ has a natural basis consisting of the nondegenerate singular $n$-cubes of $A$, where $A$ is any topological space. Let $\alpha, \beta$ be the system of degeneracies described in 1.2, and define $\chi: C \rightarrow \hat{C}$ as follows: Suppose $u: I^{n} \rightarrow A$ is a nondegenerate $n$-cube of $A$, then if $1: I^{n} \rightarrow I^{n}$ is the identity map, we define $\chi u \in C_{n}\left(I^{n}, u\right)$ to be that element which corresponds to 1 under $i(u): C_{n}\left(I^{n}\right)$ $\rightarrow C_{n}\left(I^{n}, u\right)$. With this definition of $\chi$, we have that $C: Q \rightarrow d \mathcal{G}$ is a representable functor.

Actually it is possible to prove that the functor $C$ is representable when using the trivial system of degeneracies [4], but this representation is more difficult to handle than the one we have chosen.

1.8. Notation and Conventions. Once having chosen a system of degeneracies, there is defined a natural sub-category of $\mathfrak{T}$ such that the objects are the model objects, and the maps those of the type $\alpha(u)$. This category 
will be denoted by $\mathfrak{T}^{\alpha}$. Notice that the objects of $\mathbb{M}^{\alpha}$ are the same as the objects of $\mathbb{M}$, but there are in general fewer maps in $\mathfrak{T}^{\alpha}$ than in $\mathfrak{T}$. The fact that $\mathfrak{T}^{\alpha}$ is a category is due to the relation $\alpha(\alpha(u) \alpha(v))=\alpha(u) \alpha(v)$ which is a consequence of 1.1 Axiom 4 , and shows that the composition of two maps of the type $\alpha(u)$ is again such a map. Notice that if the system of degeneracies $\alpha, \beta$ is the trivial one, then all maps in $\mathfrak{T}^{\alpha}$ are identity maps.

1.9. Definitions. A covariant functor $K: Q \rightarrow d \varrho$ is acyclic if there exist natural transformations of functors $\eta: H_{0} K \rightarrow K_{0}$, and $U: K \rightarrow K$ of degree +1 such that if $U_{n}: K_{n} \rightarrow K_{n+1}$ is the functor determined by $U$ we have

(1) $d U_{0}=1-\eta \epsilon$, and

(2) $d U_{n}+U_{n-1} d=1$ for $n>0$, where $\epsilon: K_{0} \rightarrow H_{0} K$ is the usual natural transformation. The pair $(U, \eta)$ is a contracting homotopy for $K$. Notice that (1) implies $\epsilon=\epsilon \eta \epsilon$, and that since $\epsilon$ is onto we have that $\epsilon \eta$ is the identity of $H_{0} K$.

The functor $K$ will be said to be acyclic on models relative to the system of degeneracies $\alpha, \beta$ if $K \mid \mathfrak{T}^{\alpha}$ is acyclic. If $K(M)$ is a free module for every model $M$, and $\alpha, \beta$ is the trivial system of degeneracies this just means $H_{q} K(M)=0$ for $q>0$, and $H_{0} K(M)$ is a free module.

1.10. Remarks. If $K: Q \rightarrow d \mathcal{G}$ is a functor, and $\alpha, \beta$ is a system of degeneracies on $Q$, it is evident that there is a natural differential operator on the functor $\hat{K}$ or that $\hat{K}: Q \rightarrow d \mathcal{G}$, and that the $\Gamma: \hat{K} \rightarrow K$ is a natural transformation of functors, in particular $\Gamma d=d \Gamma$. However, even if $K$ is representable it will almost never happen that $\chi d=d \chi$. If $K$ is acyclic on models, then $\hat{K}$ is acyclic and a contracting homotopy $U$ of $K \mid \mathfrak{N}^{\alpha}$ gives rise to a contracting homotopy $U$ of $\hat{K}$.

1.11. Definitions. Let $K, L: Q \rightarrow d \mathcal{g}$ be covariant functors. The natural transformations of functors $\phi, \phi^{\prime}: K \rightarrow L$ are homotopic if there is a natural transformation of functors of degree $+1, V: K \rightarrow L$ such that $d V+V d=\phi-\phi^{\prime}$. If $T: H_{0} K\left|\mathfrak{T} \rightarrow H_{0} L\right| \mathfrak{T}$ is a natural transformation of functors, we say that a natural transformation of functors $\phi: K \rightarrow L$ is an extension of $T$ if the natural transformation of functors $\phi_{*}: H_{0} K\left|\mathfrak{T C} \rightarrow H_{0} L\right| \mathfrak{T}$ induced by $\phi$ is $T$.

1.12. Theorem. Let $K, L: Q \rightarrow d \mathcal{G}$ be covariant functors, and suppose $\alpha, \beta$ is a system of degeneracies such that $K$ is representable and $L$ is acyclic on models. Under these conditions if $T: H_{0} K\left|\mathfrak{T} \rightarrow H_{0} L\right| \mathfrak{T}$ is a natural transformation of functors there exists $\phi: K \rightarrow L$ an extension of $T$, and if $\phi^{\prime}$ is an extension of $T$, $\phi$ and $\phi^{\prime}$ are homotopic.

Proof. We will give some details of the proof of the first part of this theorem, the proof of the second part will be left to the reader.

We have that $T$ induces $T: H_{0} K \rightarrow H_{0} L$. Let $(U, \eta)$ be a contracting homotopy for $L \mid \Re^{\alpha}$, and $(U, \eta)$ the corresponding contracting homotopy for $\hat{L}$. Define $\phi_{0}=\eta T \epsilon$, and by induction $\phi_{k+1}=\Gamma U_{k} \phi_{k} d \chi$. One verifies easily that $\phi$ is an extension of $T$. 
1.13. Example. Continuing with the example of 1.2 and 1.7 , we now show that the functor $C$ defined in 1.7 is acyclic on models.

If $I^{n}$ is a standard cube, define $D: I^{n} \times I \rightarrow I^{n}$ by $D\left(t_{1}, \cdots, t_{n}, t\right)$ $=\left(t t_{1}, \cdots, t t_{n}\right)$, and define $U: Q\left(I^{n}\right) \rightarrow Q\left(I^{n}\right)$ by $U v\left(t_{1}, \cdots, t_{q+1}\right)$ $=D\left(v\left(t_{2}, \cdots, t_{q+1}\right), t_{1}\right)$ for $v: I^{q} \rightarrow I^{n}$. Since if $v$ is degenerate $U v$ is also degenerate, we have that $U$ induces $U: C\left(I^{n}\right) \rightarrow C\left(I^{n}\right)$ of degree +1 . Further, suppose $f: I^{n} \rightarrow I^{p}$ where $p \leqq n$, and $f\left(t_{1}, \cdots, t_{n}\right)=\left(t_{1}, \cdots, t_{p}\right)$. Under these conditions it is evident that $U C(f)=C(f) U$. Consequently by making a few additional mechanical calculations one verifies that $C$ is acyclic on models.

1.14. Theorem. Let $K, L: Q \rightarrow d g$ be covariant functors, and $T: H_{0} K \mid \mathfrak{T}$ $\rightarrow H_{0} L \mid$ TT a natural equivalence. Suppose further

(1) there exists a system of degeneracies $\alpha, \beta$ on $a$ such that $K$ is representable, and $K, L$ are acyclic on models, and

(2) there exists a system of degeneracies $\alpha^{\prime}, \beta^{\prime}$ on a such that $L$ is representable and $K, L$ are acyclic on models. Under these conditions there is a unique natural equivalence $\phi_{*}: H K \rightarrow H L$ induced by an extension of $T$.

This theorem is an immediate corollary of the preceding theorem.

\section{Local coefficients.}

2.1. Notations. Let $(Q, \mathfrak{T})$ denote a category with models, and $\alpha, \beta$ a system of degeneracies on this category. Suppose $K: Q \rightarrow d \mathcal{G}$ is a covariant functor, and $u: M \rightarrow A$ is an element of [TH, $A]$. In this case we shall denote by $\tau(u)$ the projection of $\widehat{K}(A)$ on $K(M, u)$ (cf. 1.4), and we shall write $\theta(u)=j(u) \tau(u)$. If $\chi: K \rightarrow \hat{K}$ is a representation, we shall write $\Psi(u)=\Gamma \tau(u) \chi$ $=K(u) \theta(u) \chi$.

2.2. Definition. Suppose $K: a \rightarrow d \mathcal{G}$ is a covariant functor, and $\chi: K \rightarrow \hat{K}$ a representation. We say that $\chi$ splits, or that $K$ is split representable if

(1) $\tau(u) \chi=\chi \Psi(u)$ for every object $A$ and $u \in S(A)$, and

(2) $\theta(u) \chi=\Psi(1(M)) \theta(u) \chi$ for every object $A$ and $u \in S(A)$.

Notice that $\Psi(1(M)) \theta(1(M)) \chi \theta(u) \chi=\theta(1(M)) \chi \theta(u) \chi$, so that condition (2) may be written

$$
\theta(u) \chi=\theta(1(M)) \chi \theta(u) \chi .
$$

2.3. Example. Continuing with the example of $1.2,1.7$, and 1.13 , we wish to show now that the representation $\chi: C \rightarrow \hat{C}$ defined in 1.13 splits.

Let $u: I^{n} \rightarrow A$ be a nondegenerate singular cube. We have that $\tau(u) \chi u$ is the element of $C\left(I^{n}, u\right)$ which corresponds to $i: I^{n} \rightarrow I^{n}$. Further if $v: I^{n} \rightarrow A$ is a different nondegenerate singular cube, $\tau(u) \chi v=0$. On the other hand $\Psi(u) u$ $=u$, and $\Psi(u) v=0$. Consequently $\chi \Psi(u) u=\tau(u) \chi u$, and $\chi \Psi(u) v=0$. This means that we have verified condition (1) on basis elements which is sufficient.

Continuing with the same notation, $\theta(u) \chi u$ is the element of $C\left(I^{n}\right)$ corresponding to $1: I^{n} \rightarrow I^{n}$, and $\theta(u) \chi v=0$. Now $\Psi(1)=K(1) \theta(1) \chi$, and therefore $\Psi(1)$ acts as the identity on the cube corresponding to $1: I^{n} \rightarrow I^{n}$ and annihilates all other singular cubes on $I^{n}$. Therefore, Condition 2 is verified. 
2.4. Proposition. If $K: Q \rightarrow d \mathcal{G}$ is a covariant functor, and $\chi: K \rightarrow \hat{K}$ a split representation, then

(1) $\Psi(u) \Psi(u)=\Psi(u)$ and $\Psi(u) \Psi(v)=0$ for $v \neq u$,

(2) setting $K_{u}(A)=\Psi(u) K(A)$, we have $K(A)=\sum_{u \in S(A)} K_{u}(A)$, and

(3) if $f: A \rightarrow B$ is a map, then $K(f) \Psi(u)=\Psi(\beta(f u)) K(f) \Psi(u)$.

2.5. Definition. A covariant functor $G: \mathfrak{N} \rightarrow \mathcal{G}^{\prime}$ will be called a local system of coefficients on $Q$ if $G(v)$ is an isomorphism whenever $v$ is nondegenerate.

A local system such that $G(v)$ is an isomorphism for every map $v$ will be called a Steenrod system.

Let $F$ be a given module; the Steenrod system such that $G(M)=F$ for every model object $M$, and $G(v)=1(F)$ for every map in $M$ will be called the constant system $F$, and a local system which is naturally equivalent to $F$ will be called a simple system with module $F$. Clearly any simple system is a Steenrod system.

2.6. Lemma. The covariant functor $G: \mathfrak{T} \rightarrow \mathrm{G}^{\prime}$ is a simple system with module $F$ if and only if for each model $M$ there is an isomorphism $\xi(M): F \rightarrow G(M)$ such that for any map $v: M \rightarrow N$ in $\mathfrak{T}$ we have $G(v)=\xi(N) \xi(M)^{-1}$.

Proof. Regarding $F$ as the constant system we can regard $\xi$ as an equivalence of functors $\xi: F \rightarrow G$. Conversely, given such an equivalence of functors, we must have $G(v) \xi(M)=\xi(N) F(v)=\xi(N)$.

2.7. Definitions. Suppose $K: \propto \rightarrow d \mathcal{G}$ is a covariant functor, $\chi: K \rightarrow \hat{K}$ a split representation, and $G$ a local coefficient system on $Q$. Define a new covariant functor $K * G: Q \rightarrow \mathcal{G}$ as follows: For any object $A$

$$
K * G(A)=\sum_{u \in S(A)} K_{u}(A) \otimes G\left(M_{u}\right)
$$

where $M_{u}$ denotes the domain of $u$. For any map $f: A \rightarrow B$ we define

$$
K * G(f) \mid K_{u}(A) \otimes G\left(M_{u}\right)=\left(K(f) \mid K_{u}(A)\right) \otimes G(\alpha(f u)) .
$$

This is legitimate, for $K(f) K_{u}(A) \subset K_{\beta(f u)}(B)$ by 2.4 , and $G(\alpha(f u)): G\left(M_{u}\right)$ $\rightarrow G\left(M_{\beta(f u)}\right)$.

If $F, G: M \rightarrow G^{\prime}$ are both local systems and $\xi: F \rightarrow G$ is a natural transformation of functors, then $\xi$ induces a natural transformation $K * \xi: K * F \rightarrow K * G$ defined by

$$
K * \xi(A) \mid K_{u}(A) \otimes F\left(M_{u}\right)=1 \otimes \xi\left(M_{u}\right) .
$$

2.8. Proposition. If $K: Q \rightarrow \mathcal{G}$ is a covariant functor which has a split representation, and $G$ is a local coefficient system on $Q$, then $K * G: Q \rightarrow \mathcal{G}$ has a split representation.

Proof. We have $\theta(u) \chi \otimes 1: K_{u}(A) \otimes G(M) \rightarrow K_{1(M)}(M) \otimes G(M)$ where $M$ is the domain of $u(2.2$, Condition 2$)$. Consequently we have a natural trans- 
formation of functors $\chi: K * G \rightarrow \widehat{K} * G$ determined by the homomorphisms $\theta(u) \otimes 1$. One verifies easily that $\chi$ is a split representation.

2.9. Remarks. Let $K \circ G: M \rightarrow G$ denote the functor defined by $K \circ G(M)$ $=K(M) \otimes G(M)$ for $M$ an object, and $K \circ G(v)=K(v) \otimes G(v)$ for $v$ a map. Define a natural transformation $T: K * G \mid \Re \rightarrow K \circ G$ by $T(M) \mid K_{u}(M)$ $\otimes G\left(M^{\prime}\right)=1 \otimes G(u)$ where $u: M^{\prime} \rightarrow M$ is nondegenerate. One verifies easily that $T$ is a natural equivalence of functors since for nondegenerate $u$, we have $G(u)$ is an isomorphism (cf. 2.5). There is a natural differential operator $d$ on $K \circ G$, and we define a differential operator $d^{\prime}$ on $K * G$ by setting $d^{\prime}=T^{-1} d T$. This enables us to define a differential operator $d^{\prime \prime}$ on $K * G$ by setting $d^{\prime \prime} \mid K * G(M, u)=i(u) d^{\prime} j(u)$. Finally using the representation $\chi: K * G \rightarrow \widehat{K} * G$ defined in 2.8, we define $d$ a differential operator by setting $d=\Gamma d^{\prime \prime} \chi$. One now verifies by some trivial but slightly tedious calculations that $d \mid \mathfrak{T}=d^{\prime}$, and that the differential operator $d$ induced in $K * G$ by $d$ in $K * G$ is just $d^{\prime \prime}$. Actually we should not call $d$ a differential operator until these verifications are made for we do not know $d^{2}=0$. However, once having verified that $d$ and $d^{\prime \prime}$ agree on $K * G$ this follows immediately from $d^{2}=d^{2} \Gamma \chi=\Gamma d^{2} \chi=0$.

Having defined a differential operator in $K * G$ we henceforth regard it as a covariant functor taking $a$ into $d g$. Further it is not difficult to see that $T: K * G \mid \mathfrak{T} \rightarrow K \circ G$ induces a natural equivalence $T_{*}: H(K * G) \mid \mathfrak{T}$ $\rightarrow H(K \circ G)$.

2.10. Proposition. If $K: Q \rightarrow d \mathcal{G}$ is a covariant functor which has a split representation and is acyclic on models, and $G$ is a local coefficient system on $Q$, then $K * G: Q \rightarrow d \mathcal{G}$ is acyclic on models.

Proof. Clearly the acyclicity of $K \mid \mathfrak{T}^{\alpha}$ implies the existence of a contracting homotopy for $K \circ G$, and this can be transferred to $(K * G) \mid \Re^{\alpha}$ by $T$.

2.11. NotATION AND DEFinition. Let $K: a \rightarrow d \varrho$ be a covariant functor, and define $G(K)=H_{0} K \mid \mathfrak{T}$. We say that $K$ is augmentable if $G(K)$ is a local system. Thus $K$ is augmentable if and only if $H_{0} K(v)$ is an isomorphism for every nondegenerate map $v$ in $\mathfrak{T}$.

2.12. Definition. A covariant functor $K: Q \rightarrow d \mathcal{G}$ is a singular functor relative to the system of degeneracies $\alpha, \beta$ if

(i) it has a split representation $\chi$,

(ii) it is acyclic on models, and

(iii) $G(K)$ is a simple system with module $\Lambda$.

2.13. Theorem. If $K, K^{\prime}: a \rightarrow d \mathcal{G}$ are singular functors relative to the system of degeneracies $\alpha, \beta$, then $H K, H K^{\prime}: Q \rightarrow \mathcal{G}$ are naturally equivalent.

The preceding follows immediately from 1.14.

2.14. Theorem. Let $\alpha, \beta$ be a system of degeneracies on $Q, G: \mathfrak{M} \rightarrow \mathcal{G}^{\prime}$ a local system, and $K: Q \rightarrow d \mathcal{Q}$ a singular functor relative to $\alpha, \beta$, then 
(i) $K * G: a \rightarrow d \mathcal{G}$ is a covariant split-representable augmentable functor which is acyclic on models, and

(ii) $G$ and $G(K * G)$ are naturally equivalent.

In view of 2.8 and 2.10 only the last part of the theorem remains to be proved. This follows simply using the following lemma.

2.15. Lemma. If $K: Q \rightarrow d \mathcal{G}$ is acyclic on models, and $G: \mathfrak{M} \rightarrow \mathcal{G}^{\prime}$ is a local system, then for $M$ an object of $\mathfrak{T}, H_{0}(K \circ G)(M)=H_{0} K(M) \otimes G(M)$, and for $f: M \rightarrow N$ a map in $\mathfrak{T}, H_{0}(K \circ G)(f)=H_{0} K(f) \otimes G(f)$.

2.16. TheOREM. Let $\alpha, \beta$ be a system of degeneracies on $a, L: a \rightarrow d \mathcal{~ a ~ c o - ~}$ variant augmentable representable functor which is acyclic on models, $G=G(L)$, and let $K: Q \rightarrow d \mathcal{G}$ be a singular functor. With these hypotheses the functors

$$
H L, H(K * G): Q \rightarrow \mathcal{G}
$$

are naturally equivalent.

3. Local coefficients on indexed categories. In the preceding section we have given a definition of local coefficients, and proved certain theorems about homology with local coefficients. However, as yet we have not made explicit the connection between our definition and the classical concept of local coefficients. This section will be devoted to remedying this deficiency. In the course of this work it is convenient to introduce the notion of an indexed category.

3.1. Definition. Let $a$ be a given category. A contravariant indexing on a consists of the following:

(i) a category $\mathscr{g}$ such that the objects are sets of elements and the mappings are ordinary mappings of sets, and

(ii) a contravariant functor $T: Q \rightarrow g$.

In other words to every object $A$ of $a$ we have assigned a set $T(A)$, and to every map $f: A \rightarrow B$ of $Q$ a map $T(f): T(B) \rightarrow T(A)$ such that

(i) if $g f$ is a map in $Q$, then $T(g f)=T(f) T(g)$, and

(ii) $T(1)=1$.

Given a contravariant indexing on $a$, we define a new category $T a$ as follows:

(i) An object of $T Q$ is a pair $(A, x)$ whure $A$ is an object of $A$ and $x \in T(A)$.

(ii) If $(A, x)$ and $(B, y)$ are objects of $T a$, a map $(f, x, y):(A, x) \rightarrow(B, y)$ is composed of a map $f: A \rightarrow B$, and two indices $x$ and $y$ such that $T(f) y=x$.

If $(Q, \mathfrak{T})$ is a category with models, then $T Q$ is a category with models, the model sub-category being denoted by $T \mathfrak{T}$. An object of $T \mathscr{T}$ is an object $(M, x)$ of $T Q$ such that $M$ is a model, and a map $(f, x, y):(M, x) \rightarrow(N, y)$ of $T \mathscr{T}$ is a map in $T Q$ such that $f$ is a map in $\mathscr{T}$.

If $\alpha, \beta$ is a system of degeneracies on $\alpha$, we define a system of degeneracies still denoted by $\alpha, \beta$ on $T a$ by setting $\alpha(u, x, y)=(\alpha(u), x, T(\beta(u)) y)$, and $\beta(u, x, y)=(\beta(u), T(\beta(u)) y, y)$. 
Notice that there is a natural covariant functor taking $T Q$ into $Q$ which sends an object $(A, x)$ into $A$, and a map $(f, x, y)$ into $f$. If $K: Q \rightarrow B$ is a covariant functor we will denote by $K: T Q \rightarrow B$ the covariant functor which is the composition of $K$ and the natural functor from $T \propto$ to $Q$. Thus $K(A, x)$ $=K(A)$, and $K(f, x, y)=K(f)$.

3.2. ExAmple. Let $a$ be the category of topological spaces and continuous maps, and let $\mathscr{g}$ be the category such that an object of $\mathscr{G}$ is the set of maps $f: A \rightarrow B$ where $A$ and $B$ are topological spaces. Denote an object of $\mathscr{g}$ by $[A, B]$. A map $(f, g):[A, B] \rightarrow[C, D]$ is a pair of maps in a such that $f: C \rightarrow A$, and $g: B \rightarrow D$. Moreover, $(f, g) h=g h f$. In other words if $h: A \rightarrow B$ is an element of $[A, B]$ then $(f, g)$ sends $h$ into the map given by the diagram

$$
\begin{aligned}
& A \stackrel{f}{\leftarrow} C \\
& h \downarrow \\
& \quad B \stackrel{g}{\rightarrow} D
\end{aligned}
$$

Now let $B$ be a fixed topological space, and define $T: Q \rightarrow g$ by $T(A)$ $=[A, B]$ for $A$ an object of $Q$, and if $f: A \rightarrow C$ is a map in $Q$, then $T(f)=(f, 1)$ : $[C, B] \rightarrow[A, B]$.

Suppose that we are given an ordinary local coefficient system in the classical sense [8] on $B$. Then every $\operatorname{map} f: A \rightarrow B$ induces such a system on $A$. Consequently every object of the category $T Q$ has attached to it a classical coefficient system. Moreover, since cubes are simply connected, the objects $\left(I^{n}, f\right)$ where $f: I^{n} \rightarrow B$ have attached to them a unique group. In other words if we consider $Q$ as a category with models as in the earlier examples, we now have a local coefficient system such as we have described earlier on the category $T a$.

We now pass on to show the connection between local coefficients as we have defined them, and as defined classically [8] in a more abstract manner.

Assumption. $(Q, \mathfrak{T}, \alpha, \beta)$ is a fixed category with models and degeneracies, and $T: Q \rightarrow \mathscr{g}$ is a contravariant indexing on $\alpha$. This hypothesis will be retained for the remainder of this section.

3.3. Proposition. If $K: a \rightarrow d g$ is a covariant functor which is split representable, acyclic on models, or singular, then so is $K: T Q \rightarrow d G$.

Model assumptions I. We assume there are three special models, $P, I$ and $I^{2}$ called respectively the point, the interval, and the square. Further, we assume these models satisfy the conditions listed below.

(1) For every object $A$ in $a$ there is a unique map of $A$ into $P$.

(2) For every object $A$ there is a map $u: P \rightarrow A$, and if $u$ is any such map $\alpha(u)=1$. 
(3) There are maps $j^{0}, j^{1}: P \rightarrow I$ and nondegenerate maps $k^{0}, k^{1}, k^{2}: I \rightarrow I^{2}$ all of which lie in $\mathbb{M}$ and such that

$$
\begin{aligned}
& k^{0} j^{1}=k^{1} j^{0}, \\
& k^{0} j^{0}=k^{2} j^{0}, \\
& k^{2} j^{1}=k^{1} j^{1} .
\end{aligned}
$$

3.3'. EXAmple. Consider again the category of topological spaces and continuous maps. $P$ is the point, $I$ the unit interval, and $I^{2}$ the square. Define $j^{0}(P)=0, j^{1}(P)=1$. The maps $k^{0}, k^{1}, k^{2}$ are maps which go around the edges of the square.

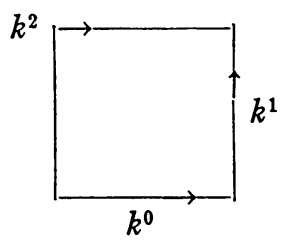

We define $k^{0}(t)=(t, 0), k^{1}(t)=(1, t), k^{2}(t)=(0,2 t)$ for $0 \leq t \leq 1 / 2$, and $k^{2}(t)$ $=(1,2 t-1)$ for $1 / 2 \leq t \leq 1$.

3.4. Definition. A map of $P$ into $A$ is called a point of $A$, and a map $u: I \rightarrow A$ is called a path in $A$. The path $u$ has initial point $u j^{0}$, final point $u j^{1}$, and is said to be a path connecting these points. Two paths $v, w: I \rightarrow A$ are homotopic if there exist maps $u: I^{2} \rightarrow A$ and $f: P \rightarrow A$ such that $u k^{0}=v$, $u k^{2}=w$, and $u k^{1}=f g$ where $g: I \rightarrow P$ is the unique map. Notice that if $v, w$ are homotopic they have the same end points.

An object $A$ is connected if every pair of points of $A$ can be joined by a path, and is simply-connected if any two paths with the same end points are homotopic.

Notation. If $u: I \rightarrow A$ is a path let $[u]$ denote the homotopy class of $u$.

Model assumptions II.

(1) The model objects in $a$ are connected and simply connected.

(2) Let $u, v: I \rightarrow A$ be paths.

(i) If the final point of $u$ is the initial point of $v$, then there is a map $w: I^{2} \rightarrow A$ such that $w k^{0}=u$ and $w k^{1}=v$.

(ii) If the final point of $u$ is the final point of $v$, then there is a map $w: I^{2} \rightarrow A$ such that $w k^{1}=u$ and $w k^{2}=v$.

(iii) If the initial point of $u$ is the initial point of $v$, then there is a map $w: I^{2} \rightarrow A$ such that $w k^{0}=u$ and $w k^{2}=v$.

(3) Let $u, v, w: I \rightarrow A$ be paths such that $u j^{1}=v j^{0}, v j^{1}=w j^{0}$. Let $F, F^{1}$ : $I^{2} \rightarrow A$ be such that $F k^{0}=u, F k^{1}=v, F^{1} k^{0}=v, F^{1} k^{1}=w$, and let $f, f^{1}: I^{2} \rightarrow A$ be such that $f k^{0}=F k^{2}, f k^{1}=v, f^{1} k^{0}=u, f^{1} k^{1}=F^{1} k^{2}$. In this case $\left[f k^{2}\right]=\left[f^{1} k^{2}\right]$.

Condition 1 in the preceding is self explanatory. Condition 2 says that if you have a map defined on a closed connected part of the boundary of a 
square which is not the whole boundary, you can extend to a map of the square. Geometrically this is evident since the square has such a piece of its boundary as a retract. This condition (a form of the extension condition of Kan applied to squares) allows one to define a product between paths $u$ and $v$ such that the final point of $u$ is the initial point of $v$. Condition 3 then says that this product is associative. With these axioms it is clearly possible to define the fundamental group of an object $A$ in $Q$ based at any point of $A$.

3.5. Definitions. For each model object $M$ choose a map $j(M): P \rightarrow M$, suppose $j(I)=j^{0}$, and $j\left(I^{2}\right)=k^{0} j^{0}$.

If $G: T \mathfrak{T} \rightarrow \mathcal{G}^{\prime}$ is a local system define a new covariant functor $G^{R}: T \mathfrak{T} \rightarrow \mathcal{G}^{\prime}$ as follows:

(1) If $M$ is a model object and $x \in T(M), G^{R}(M, x)=G(P, T(j(M)) x)$.

(2) If $(f, x, y):(M, x) \rightarrow(N, y)$ is a map in $T M, G^{R}(f, x, y)=(G,(j(N)$, $z, y))^{-1} G(f, x, y) G\left(j(M), z^{\prime}, x\right)$ where $z=T\left(j(N) y\right.$ and $z^{\prime}=T(j(M)) x$.

3.6. Lemma. The transformation $T_{G}: G \rightarrow G^{R}$ defined by $T_{G}(M, x)$ $=\left(G(j(M), T(j(M)) x, x)^{-1}\right.$ is a natural equivalence, and $G^{R}$ is a local system.

3.7. Definition. $G: T \mathfrak{T} \rightarrow \mathcal{G}^{\prime}$ is a reduced local system if $T_{G}$ is the identity.

3.8. Lemma. If $G: T \mathfrak{T} \rightarrow \mathcal{G}^{\prime}$ is a local system, then $G^{R}: T \mathfrak{N} \rightarrow \mathrm{G}^{\prime}$ is a reduced local system.

Assumption. $G: T \mathfrak{T} \rightarrow \mathrm{G}^{\prime}$ is a reduced local system which we consider fixed.

3.9. Definition. If $w: I \rightarrow A$ is a path in $A$, and $x \in T(A)$, we define

$$
\xi_{w, x}=G\left(j^{1}, T\left(w j^{1}\right) x, T(w) x\right): G\left(P, T\left(w j^{1}\right) x\right) \rightarrow(P, T(w) x) .
$$

Since $G$ is a reduced system $G\left(P, T\left(w j^{0}\right) x\right)=G(P, T(w) x)$, and therefore

$$
\xi_{w, x}: G\left(P, T\left(w j^{1}\right) x\right) \rightarrow G\left(P, T\left(w j^{0}\right) x\right) .
$$

In other words given a path $w$ connecting two points of $A \xi_{w, x}$ is an isomorphism of the group attached to one point with the group attached to the other.

3.10. Lemma. If $u, v: I \rightarrow A$ are homotopic paths then $\xi_{u, x}=\xi_{v, x}$.

3.11. Lemma. Let $f: M \rightarrow N$ be a map in $\mathfrak{T}$, and let $z \in T(N)$. Then $G(f, T(z)$, $z)=\xi_{w, y}$ where $w$ is any path in $N$ joining $j(N)$ and $f j(M)$.

3.12. Lemma. If $u, v, w: I \rightarrow A$ are paths such that there exists a map $F: I^{2} \rightarrow A$ with $F k^{0}=u, F k^{1}=v, F k^{2}=w$ and $x \in T(A)$, then

$$
\xi_{w, x}=\xi_{u, x} \xi_{v, x} .
$$

3.13. Definition. A path $u: I \rightarrow A$ is the constant path at $a=u j^{0}$ if $u=u j^{0} g$ where $g: I \rightarrow P$ is the unique map. 
3.14. Lemma. If $u: I \rightarrow A$ is the constant path at some point a of $A$ and $x \in T(A)$, then

$$
\xi_{u, x}=1 \text {. }
$$

3.15. Definition. Let $A$ be an object of $A$. A classical local coefficient system $(G, \xi)$ on $A$ is the assignment to each point $a$ of $A$ of a module $G(a)$, and to each path $u$ of $A$ with initial point $a$ and final point $b$ an isomorphism $\xi_{u}: G(b) \rightarrow G(a)$ such that

(i) if $u$ and $v$ are homotopic then $\xi_{u}=\xi_{v}$,

(ii) if $u$ is the constant path at $a \in A, \xi_{u}=1$, and

(iii) if $u, v, w$ are paths in $A$ such that there is a map $F: I^{2} \rightarrow A$ and $F k^{0}=u, F k^{1}=v, F k^{2}=w$, then $\xi_{w}=\xi_{u} \xi_{v}$.

3.16. Proposition. Let $G: T \mathfrak{T} \rightarrow \mathcal{G}^{\prime}$ be a reduced local system, $A$ an object of $a$, and $x \in T(A)$. Then if one defines

$$
\begin{aligned}
G x(a) & =G(P, T(a) x) \text { for } a \in A, \text { and } \\
\xi x(u) & =\xi_{u, x} \text { for } u \text { a path in } A,
\end{aligned}
$$

$(G x, \xi x)$ is a classical local coefficient system on $A$.

3.17. Proposition. Any local system $G: T \mathfrak{T} \rightarrow \mathcal{G}^{\prime}$ is a Steenrod system.

Remarks. Suppose that $A$ is the category of topological spaces and continuous maps, and that the contravariant indexing is as in Example 3.2. Let $(G, \xi)$ be a classical local coefficient system on the fixed space $B$. If $(M, u)$ is an object of $T$ TI, define $G^{\prime}(M, u)=G(u j(M))$, and if $(f, u, v):(M, u) \rightarrow(N, v)$ is a map in $T \mathscr{T}$, define $G^{\prime}(f, u, v)=\xi_{w}$ where $w: I \rightarrow N$ is any path from $v j(N)$ to $v f j(M)=u j(M)$. In this case $G^{\prime}: T \Re\left(\rightarrow \mathcal{G}^{\prime}\right.$ is a local system.

\section{The spectral Sequence of a map}

\section{Algebraic concepts.}

1.1. Definition. Let $A$ be a differential graded module, i.e. an object of $d g$. The module $A$ is said to be filtered or have a filtration if there is given a submodule $F_{p} A$ for every integer $p$ such that

(1) $F_{p} A=0$ for $p<0$,

(2) $F_{p} A \subset F_{p+1} A$,

(3) $d F_{p} A \subset F_{p} A$, and

(4) $A_{n}=\sum_{p} F_{p} A \cap A_{n}$.

The set $\left\{F_{p} A\right\}$ is said to be a filtration on $A$. Let $f: A \rightarrow B$ be a map of differential graded modules, and suppose both $A$ and $B$ are filtered. The map $f$ is filtration preserving if $f\left(F_{p} A\right) \subset F_{p} B$. We denote by $d \mathcal{F}$ the category of differential graded filtered modules and filtration preserving maps.

There are several functors mapping the category $d \mathcal{F}$ into the category 
$d g$. First there is the functor which neglects the filtration. Secondly the theory of spectral sequences (cf. [2] and [3]) provides us with a sequence of functors

$$
E^{r}: d \mathfrak{F} \rightarrow d \mathcal{G}
$$

such that $H E^{r}=E^{r+1}$. Our notation is a compromise between that of Serre, and that of Cartan and Eilenberg. For all definitions and proofs we refer to [3]. We shall write $E_{p, q}^{r}(A)$ for the bigraded spectral sequence of $A$, so that $d^{r}: E_{p, q}^{r} \rightarrow E_{p-r, q+r-1}^{r}$ where $p$ is the filtration degree, and $p+q$ the total degree or dimension. Recall that $E_{p, q}^{0}=F_{p} A \cap A_{p+q} / F_{p-1} A \cap A_{p+q}$.

1.2. Lemma. If $f, g: A \rightarrow B$ are maps in $d \mathcal{F}$ and $V: A \rightarrow B$ is a homomorphism of modules such that

(1) $V A_{n} \subset B_{n+1}$,

(2) $V F_{p} A \subset F_{p+s} B$ for a fixed $s$, and

(3) $f-g=d V+V d$

then $V$ induces a homomorphism $V^{s}: E^{s}(A) \rightarrow E^{s}(B)$ such that

$$
\begin{aligned}
& V^{s} E_{p, q}^{s}(A) E_{p+s, q-s+1}^{s}(B), \text { and } \\
& E^{s}(f)-E^{s}(g)=d^{s} V^{s}+V^{s} d^{s} .
\end{aligned}
$$

The proof of this lemma may be found in [5].

COROLlaRY. Under the conditions of the preceding lemma

$$
E^{r}(f)=E^{r}(g) \text { if } r>s .
$$

Clearly the homomorphism $V$ described above is a homotopy. Such a homotopy is said to be of degree $s$, and as we have seen above induces a homotopy at the stage $s$ of the spectral sequences involved.

1.3. Notation. If $A$ is a category, $K, L: Q \rightarrow d \mathcal{F}$ are covariant functors, and $T: K \rightarrow L$ is a natural transformation of functors, then $T$ induces natural transformations $T^{r}: E^{r} K \rightarrow E^{r} L(r \geq 0)$.

2. Categories with dimension.

2.1. Definitions. We say that $(Q, \mathfrak{T}, \gamma)$ is a category with models and dimension if

(1) $(Q, \mathfrak{T})$ is a category with models, and

(2) $\gamma:[\Re, a] \rightarrow Z^{+}$(the non-negative integers) is a function such that $\gamma(u)=\gamma\left(1_{M}\right)$ where $u: M \rightarrow A$ is a map in $A$, and $1_{M}: M \rightarrow M$ is the identity map of the model $M$.

A system of degeneracies $\alpha, \beta$ on $Q$ is compatible with dimension if

(1) $\gamma(\beta(u)) \leq \gamma(u)$, and

(2) $\gamma(\beta(u))=\gamma(u)$ implies $\alpha(u): M \rightarrow M$ is the identity where $M$ is the domain of $u$.

The system of degeneracies $\alpha, \beta$ is weakly compatible with dimension if

(1) $\gamma(\beta(u)) \leq \gamma(u)$. 
Assumption. $(\mathfrak{Q}, \mathfrak{T}, \boldsymbol{\gamma})$ is a category with models and dimension which is fixed for the rest of this section.

2.2. Definitions. Suppose $\alpha, \beta$ is a system of degeneracies on $Q, K$ : $a \rightarrow d \mathcal{G}$ is a covariant functor, and $\chi: K \rightarrow \hat{K}$ a split representation. We say that $\chi$ is weakly compatible with dimension if

(1) $K_{n}(A) \cap K_{u}(A) \neq 0$ implies $\gamma(u) \leq n$, and that $\chi$ is compatible with dimension if it satisfies (1), and

(2) $\Psi(v) d \Psi(u) \neq 0$ implies $\gamma(v)<\gamma(u)$ or $v=u$.

The representation $\chi$ is normalized if $K_{n}(A) \cap K_{u}(A) \neq 0$ implies $\gamma(u)=n$. Notice that in this case $\Psi(v) d \Psi(u) \neq 0$ implies $\gamma(v)=\gamma(u)-1$, so that $\chi$ is compatible with dimension.

2.3. Definitions. Let $\alpha, \beta$ be a system of degeneracies on $a$ which is weakly compatible with dimension, $K: a \rightarrow d g$ a covariant functor, and $\chi: K \rightarrow \hat{K}$ a split representation which is weakly compatible with dimension. For any object $A$, let

$$
S^{p}(A)=\{u \mid u \in S(A) \text { and } \gamma(u) \leq p\} .
$$

Define $F_{p} K(A)=\sum_{u \in S^{p}(A)} K_{u}(A)$, and define $F_{p} \hat{K}(A)=\sum_{u \in S(A)} F_{p} K(M, u)$ where $F_{p} K(M, u)$ is naturally isomorphic with $F_{p} K(M)$.

2.4. Proposition. With the hypotheses of $2.3, K, \hat{K}: Q \rightarrow d F, \Gamma: \hat{K} \rightarrow K$ is a natural transformation in $d \mathcal{F}$, and $\chi: K \rightarrow \hat{K}$ is filtration preserving. Further $\chi$ induces $\chi^{0}: E^{0} K \rightarrow E^{0} \hat{K}=\widehat{E}^{0} K, \chi^{0}$ is a split representation, and $\Psi^{0}(v) E_{p, q}^{0} K(A)$ $\neq 0$ implies $\gamma(v)=p$.

The proof of this proposition is a routine verification.

2.5. Proposition. If in addition to the hypotheses of 2.3 , the system of degeneracies $\alpha, \beta$ is compatible with dimension, and the representation $\chi$ is compatible with dimension, then

(1) $d^{0} \chi^{0}=\chi^{0} d^{0}$, and

(2) $\chi^{0}$ induces a split representation $\chi^{1}: E^{1} K \rightarrow E^{1} K=E^{1} K$.

Proof. Part 2 of the proposition follows immediately from part 1 . To prove part 1, let $A$ be an object and $u$ an element of $S(A)$ such that $\gamma(u)=p$. We will write $\equiv$ for equality modulo elements of filtration $<p$. Now our result will follow if we can prove $d \chi(A) \Psi(u) \equiv \chi(A) d \Psi(u)$, where $u=\beta(u)$ and $\alpha(u)$ is the identity map $1(M): M \rightarrow M$ of the domain of $u$. By 2.2, 2, we have $(1-\Psi(1(M))) d \Psi(1(M)) F_{p} K(M) \subset F_{p-1} K(M)$, and hence $d \Psi(1(M))$ $\equiv \Psi(1(M)) d \Psi(1(M))$. Therefore

$$
\begin{aligned}
d \theta(u) \chi(A) & =d \Psi(1(M)) \theta(u) \chi(A) \\
& \equiv \Psi(1(M)) d \theta(u) \chi(A) \\
& =\theta(1(M)) \chi(M) d \theta(u) \chi(A)
\end{aligned}
$$


and

$$
\begin{aligned}
\chi(A) d \Psi(u) & =\chi(A) d K(u) \theta(u) \chi(A) \\
& =\chi(A) K(u) d \theta(u) \chi(A) \\
& \equiv \hat{K}(u) \chi(M) \Psi(1(M)) d \theta(u) \chi(A) \\
& =\hat{K}(u) \tau(1(M)) \chi(M) d \theta(u) \chi(A) \\
& =i(u) j(1(M)) \tau(1(M)) \chi(M) d \theta(u) \chi(A) \\
& =i(u) \theta(1(M)) \chi(M) d \theta(u) \chi(A) \\
& \equiv i(u) d \theta(u) \chi(A) \\
& =d \tau(u) \chi(A) \\
& =d \chi(A) \Psi(u),
\end{aligned}
$$

and the proof is complete.

The preceding proposition is a basic step in our calculation of $E^{2}$ in the spectral sequence for a fibre space. It is part of what enables us to calculate $E^{2}$ without actually calculating $E^{1}$. This will not be used until later. At present we content ourselves with giving an application showing the existence of normalized functors.

2.6. Definition. Suppose the hypotheses of 2.3 are satisfied. For each $q \geq 0$ define $E_{*, q}^{1} K: a \rightarrow d \varrho$ by

$$
E_{*, q}^{1} K(A)=\sum_{p \geq 0} E_{p, q}^{1} K(A),
$$

and

$$
E_{*, q}^{1} K(f)=\sum_{p \geq 0} E_{p, q}^{1} K(f)
$$

where $A$ is an object of $Q$, and $f$ is a map. The differential operator is that induced by $d^{1}: E_{p, q}^{1} K(A) \rightarrow E_{p-1, q}^{1} K(A)$, and the gradation is defined by $\left(E_{*, q}^{1} K(A)\right)_{p}=E_{p, q}^{1} K(A)$.

2.7. TheOREm. Let $\alpha, \beta$ be a system of degeneracies on $a$ which is weakly compatible with dimension, $K: Q \rightarrow d g$ a covariant functor, and $\chi: K \rightarrow \hat{K}$ a split representation which is compatible with dimension. Then for each $q \geq 0$, the functor $E_{*, q}^{1} K: Q \rightarrow d \mathcal{G}$ has a normalized split representation induced by $\chi$. If further $K$ is acyclic on models by means of a contracting homotopy $U$ such that

$$
U F_{p} K\left|\mathfrak{T} \subset F_{p+1} K\right| \mathfrak{T},
$$

and $L=E_{*, 0}^{1}$, then $H K$ and $H L$ are naturally equivalent.

\section{3 . Induced structures.}

3.1. Definition. Let $a$ be the category of topological spaces and continuous maps. Suppose $f: A \rightarrow B$ and $\pi: E \rightarrow B$ are maps. Define $R(f, \pi)$ 
$=\{(a, e) \mid a \in A, e \in E, f(a)=\pi(e)\}$ topologized as a subspace of $A \times E$. Define

$$
Q(f, \pi): R(f, \pi) \rightarrow E \quad \text { by } Q(f, \pi)(a, e)=e,
$$

and

$$
P(f, \pi): R(f, \pi) \rightarrow B \quad \text { by } \quad P(f, \pi)(a, e)=a .
$$

The preceding gives rise to a commutative diagram

$$
\begin{array}{rlrl}
R(f, \pi) \stackrel{Q(f, \pi)}{\longrightarrow} & E \\
P(f, \pi) \quad \downarrow & & \downarrow \pi \\
A & \quad & \downarrow
\end{array}
$$

The space $R(f, \pi)$ is the space induced by the map $f$ from the map $\pi$. In this section we abstract this process so as to be able to deal with it in fairly general categories. Though we do not use the fact in this paper, the abstraction of induced space works as well for semi-simplicial complexes and semi-simplicial maps, or for semi-cubical complexes and semi-cubical maps as it does for topological spaces and continuous maps.

3.2. Definition. Given a category $a, \tilde{a}$ will denote the category defined as follows:

The objects are ordered pairs of maps of $a$ with a common range; the object $A \stackrel{f}{\rightarrow} B \stackrel{\bullet}{\leftarrow} C$ will be denoted by $(f, g)$.

The maps are commutative diagrams of $A$ such as

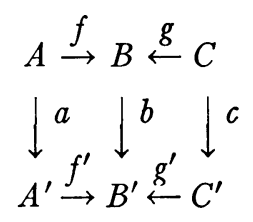

which will be denoted by $\left(a, b, c ; f, g ; f^{\prime}, g^{\prime}\right)$, or sometimes ambiguously by $(a, b, c)$. The domain of the map above is $(f, g)$ and the range $\left(f^{\prime}, g^{\prime}\right)$.

3.3. Definition. Given a category $Q$, a covariant functor $\Sigma: \widetilde{a} \rightarrow D D Q$ (the derived category of the derived category of $a$, cf. chapter 0 ) denoted by $\Sigma=\Sigma(P, Q, R, S)$ is an induced structure on $a$ if it satisfies the following axioms:

(1) For an object $(f, \pi)$ of $\tilde{a},(f, \pi)$ is the object $(Q(f, \pi), f ; P(f, \pi), \pi)$ of $D D Q$, i.e. the commutative diagram

$$
\begin{array}{rl}
R(f, \pi) \stackrel{Q(f, \pi)}{\longrightarrow} & E \\
P(f, \pi) \downarrow & \\
A & \downarrow \pi \\
A & B
\end{array}
$$

in $a$. 
(2) For a map $\left(a, b, e ; f, \pi ; f^{\prime}, \pi^{\prime}\right)$ of $\tilde{Q}, \Sigma(a, b, e)$ is the mapping of $D D Q$ shown in the diagram below

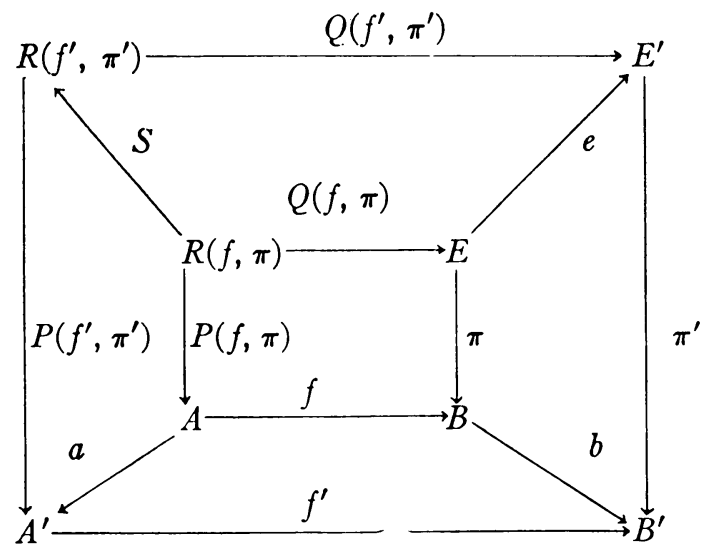

in which $S=S\left(a, b, e ; f, \pi ; f^{\prime}, \pi^{\prime}\right)$.

(3) Given $(f, \pi)$ in $\widetilde{a}$ and $h, h^{\prime}: X \rightarrow R(f, \pi)$, maps in $a$ such that

$$
P(f, \pi) h=P(f, \pi) h^{\prime},
$$

and

$$
Q(f, \pi) h=Q(f, \pi) h^{\prime}
$$

then $h=h^{\prime}$.

(4) Given a map $\pi: E \rightarrow B$ of $Q$, there is a map $\sigma(\pi): E \rightarrow R(\pi, \pi)$ such that

$$
P(\pi, \pi) \sigma(\pi)=Q(\pi, \pi) \sigma(\pi)=1(E) .
$$

This last hypothesis assures the existence of certain cross sections.

3.4. Lemma. Given maps $\stackrel{g}{\rightarrow} \underset{E \rightarrow}{\rightarrow} B$ of $a$, there is a unique map $\sigma(\pi, g)$ : $A \rightarrow R(\pi g, \pi)$ such that

$$
\begin{aligned}
& P(\pi g, \pi) \sigma(\pi, g)=1(A), \\
& Q(\pi g, \pi) \sigma(\pi, g)=g .
\end{aligned}
$$

Moreover, we have that $\sigma(\pi, 1(E))=\sigma(\pi)$.

Proof. Let $\sigma(\pi, g)=S(1(A), 1(B), g ; \pi g, \pi g ; \pi g, \pi) \sigma(\pi g)$. One verifies readily that this map satisfies the conditions of the lemma, and uniqueness follows from 3.2 , (3).

3.5. Lemma. Given a map $\pi: E \rightarrow B$ in $Q$, there is a map $q^{\prime}(\pi): E \rightarrow R(1(B), \pi)$ such that if $q(\pi)=Q(1(B), \pi)$, then

$$
q(\pi) q^{\prime}(\pi)=1,
$$


and

$$
q^{\prime}(\pi) q(\pi)=1
$$

Proof. Let $q^{\prime}(\pi)=S(\pi, 1(B), 1(E) ; \pi, \pi ; 1(B), \pi) \sigma(\pi)$.

3.6. Definition. If $\Sigma=\Sigma(P, Q, R, S)$ is a given induced structure on $a$ we define a new induced structure $\bar{\Sigma}=\bar{\Sigma}(\bar{P}, \bar{Q}, \bar{R}, \bar{S})$ on $Q$ as follows:

For an object $(f, \pi)$ of $\bar{a}, \pi: E \rightarrow B$, we define

$$
\bar{\Sigma}(f, \pi)=\Sigma(f, \pi) \text { if } f \neq 1(B),
$$

and

$$
\bar{\Sigma}(f, \pi)=(1(E), 1(B) ; \pi, \pi) \text { if } f=1(B) .
$$

For a map $\left(a, b, e ; f, \pi ; f^{\prime}, \pi^{\prime}\right)$ define $\bar{\Sigma}$ of this map by replacing $P, Q, R, S$ in the preceding diagram by $\bar{P}, \bar{Q} ; \bar{R}, \bar{S}$ where

$$
\begin{aligned}
\bar{S} & =S=S\left(a, b, e ; f, \pi ; f^{\prime}, \pi^{\prime}\right) & & \text { if } f \neq 1(B), f^{\prime} \neq 1\left(B^{\prime}\right), \\
& =q\left(\pi^{\prime}\right) S & & \text { if } f \neq 1(B), f^{\prime}=1\left(B^{\prime}\right), \\
& =S q^{\prime}(\pi) & & \text { if } f=1(B), f^{\prime} \neq 1\left(B^{\prime}\right), \\
& =q\left(\pi^{\prime}\right) S q^{\prime}(\pi) & & \text { if } f=1(B), f^{\prime}=1\left(B^{\prime}\right),
\end{aligned}
$$

using the notation of 3.5 .

Finally we define a natural equivalence $T: \Sigma \rightarrow \bar{\Sigma}$ by

where

$$
T(f, \pi)=(t(f, \pi), 1(A), 1(B), 1(E))
$$

$$
A \stackrel{f}{\rightarrow} B \stackrel{\pi}{\leftarrow} E
$$

is an object of $\tilde{a}$, and

$$
t(f, \pi): R(f, \pi) \rightarrow \bar{R}(f, \pi)
$$

is given by

$$
\begin{array}{ll}
t(f, \pi)=1 & \text { if } f \neq 1(B), \\
t(f, \pi)=q(\pi) & \text { if } f=1(B),
\end{array}
$$

again using the notation of 3.5 .

An induced structure $\Sigma$ is said to be reduced if $T: \Sigma \rightarrow \bar{\Sigma}$ is the identity.

3.7. Lemma. If $\Sigma$ is any induced structure, then $\bar{\Sigma}$ is a reduced structure.

Assumption. $(Q, \mathfrak{T})$ is a category with models, and $\Sigma=\Sigma(P, Q, R, S)$ is a reduced induced structure on $Q$.

Thus, given $\pi: E \rightarrow B$ we have $R(1(B), \pi)=E, q(\pi)=Q(1(B), \pi)=1(E)$, $P(1(B), \pi)=\pi, \sigma(1(E))=1(E)$.

Our aim is now to show how to turn the category $D Q$ into a category with models (DQ, DIT), and to show how a system of degeneracies $\alpha, \beta$ on $Q$ 
gives rise naturally to a system of degeneracies on $D Q$. Further we will show how a dimension function $\gamma$ on $Q$ gives rise to dimension functions on $D Q$. We will carry out this process using the induced structure $\Sigma$. One could turn $D Q$ into a category with models without using $\Sigma$, but the models would be different. This would be done by saying that a model in $D Q$ is a map in $\mathfrak{T}$, and a map in $\mathscr{D T}$ a commutative square diagram in $\mathfrak{T}$, in other words by letting the model sub-category of $D Q$ be the derived category of $\mathfrak{T}$. For some purposes this would seem to be the most convenient way to define a model structure on $D Q$, but we have not been able to handle this structure very well. If we had a dimension function $\gamma$ on $\alpha$, and a system of degeneracies $\alpha, \beta$ on $\alpha$, which was compatible with dimension, the preceding would give rise to a dimension function $\gamma$ on $D Q Q$ as follows: Suppose $u: M \rightarrow M^{\prime}$ is a map in $\mathfrak{T}$, this is an object of $D \mathscr{T}$ and we would define $\gamma(1(u))=\gamma\left(M^{\prime}\right)$, thus completely determining the dimension function on DIT. If

$$
\begin{gathered}
M \stackrel{f}{\rightarrow} N \\
u \downarrow \\
M^{\prime} \stackrel{g}{\rightarrow} N^{\prime}
\end{gathered}
$$

is a map in $D \mathscr{T}$, and we tried to define $\alpha$ of this map to be

$$
\begin{aligned}
& M \stackrel{\alpha(f)}{\longrightarrow} M^{\prime \prime} \\
& \downarrow u \quad \downarrow^{\alpha} \quad{ }^{\alpha}(v(f)) \\
& M^{\prime} \stackrel{\alpha(g)}{\longrightarrow} M^{\prime \prime}
\end{aligned}
$$

we would not in general have a commutative diagram. If, however, $\beta(u)$ $=1\left(M^{\prime}\right)$ then the diagram would be commutative, so if we restricted our models in $D Q$ to be those maps which were in $\Re^{\alpha}$, we would be all right at this stage. Further the definition of $\beta$ by the diagram

$$
\begin{array}{ll}
N^{\prime \prime} \stackrel{\beta(f)}{\longrightarrow} & N \\
\downarrow \alpha(v \beta(f)) \quad & \downarrow v \\
\downarrow & \stackrel{\beta(v f)}{\longrightarrow} N^{\prime}
\end{array}
$$

would give us a system of degeneracies on the models sub-category of $D Q$. This system of degeneracies would be weakly compatible with dimension, but not compatible with dimension.

Now a functor $K: Q \rightarrow d \varrho$ which was representable by a representation $\chi$ which was compatible with dimension would give rise to a functor $K: D Q \rightarrow d \mathcal{G}$ which would be representable by a representation $\chi$ which would be weakly compatible with dimension. It would seem reasonable that two singular 
functors on $a$ which had contracting homotopies of degree 1 on the model derived category would give rise to the same spectral sequences from $E^{2}$ on. We have been unable to prove this. Consequently we now abandon this procedure, and continue our program with the aid of an induced structure.

3.8. Definitions. We define DT to be the category such that objects of $D \mathscr{T}$ are maps in $a$ whose range is an object of $\mathscr{M}$, and such that maps are commutative diagrams

$$
\begin{aligned}
A \stackrel{g}{\rightarrow} B \\
u \downarrow \\
M \stackrel{f}{\rightarrow} N
\end{aligned}
$$

in $a$ where $f$ is a map in IT. If $\alpha, \beta$ is a system of degeneracies on $\alpha$, we define $\alpha(g, f ; u, v)=(h, \alpha(f), u ; P(\beta(f), v))$, and $\beta(g, f ; u, v)=(Q(\beta(f), v), \beta(f)$; $P(\beta(f), v) v)$, where $h=S(\alpha(f) u, 1(N), g ; f u, f u ; \beta(f), v)$. This is clearer if one looks at the diagram

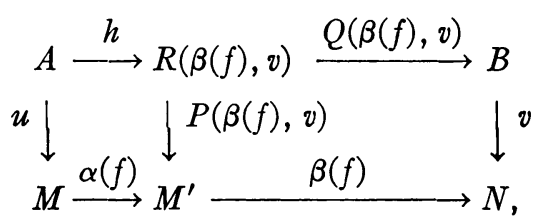

and recalls that $Q(\beta(f), v) h=g$.

Notice that in the preceding we have a strong violation of notation in that DTI is not the derived category of $\mathfrak{T}$.

3.9. Proposition. (DQ, DTC) is a category with models, and a system of degeneracies $\alpha, \beta$ on a gives rise to one on $D Q$ (cf. 3.8).

The proof of this proposition is a bit tedious, but not difficult.

3.10. Definition. If $(\propto, \mathfrak{T}, \gamma)$ is a category with models and dimension, define $\gamma:$ DTT $\rightarrow Z^{+}$by

$$
\gamma(1(u))=\gamma(1(M))
$$

where $u: A \rightarrow M$, and $M$ is a model object.

3.11. THEOREM. If ( $\alpha, \mathfrak{T}, \alpha, \beta, \gamma)$ is a category with models, dimension, and degeneracies, then (DQ, DMC, $\alpha, \beta, \gamma)$ is a category with models, dimension, and degeneracies. Further, if the system of degeneracies on $a$ is weakly compatible with dimension, so is the one on DQ.

4. Functors on DQ.

Assumption. Throughout this section $(\alpha, \mathfrak{T}, \alpha, \beta, \gamma)$ is a given category with models, degeneracies, and dimension, and the system of degeneracies 
$\alpha, \beta$ is weakly compatible with dimension. Moreover, $\Sigma=\Sigma(P, Q, R, S)$ is a reduced structure on $Q$, and (DQ, DMT, $\alpha, \beta, \gamma)$ is the category with models, degeneracies, and dimension, defined in 3.8.

We will also keep fixed the following notations: $\pi: E \rightarrow B, \pi^{\prime}: E^{\prime} \rightarrow B^{\prime}$ are maps of $Q, F=\left(f, g ; \pi, \pi^{\prime}\right)$ is a map of $D Q$.

4.1. Definitions. Let $K: a \rightarrow g$ be a covariant functor. Define covariant functors

$$
K^{D}: D Q \rightarrow G, \quad \text { and } \quad K^{R}: D Q \rightarrow G
$$

by $K^{D}(\pi)=K(E), K^{D}(F)=K(f) ; K^{R}(\pi)=K(B), K^{R}(F)=K(g)$.

In other words $K^{D}$ is the functor corresponding to the domain of objects in $D Q$ when they are considered as maps in $Q$, and $K^{R}$ is the functor corresponding to the range of similar objects. If $K: a \rightarrow d \mathcal{G}$, then in an obvious way $K^{D}$ and $K^{R}$ may also be regarded as functors into $d \mathcal{~}$.

Notations. We will write $\hat{K}^{D}$ and $\hat{K}^{R}$ for the $\wedge$ functor on $D Q$ corresponding to $K^{D}$ and $K^{R}$. Further to shorten notation in this section we will write $v^{\pi}=\Sigma(v, \pi)$.

4.2. Definition. Let $K: a \rightarrow d g$ be a covariant functor, and $\chi: K \rightarrow \hat{K}$ be a split representation. Define $\chi: K^{R} \rightarrow \hat{K}^{R}$ by

$$
\chi(\pi) \mid K_{v}(B)=i\left(v^{\pi}\right) \theta(v) \chi(B) .
$$

Note that in the preceding definition we are using the fact that $\chi$ is a split representation.

4.3. Proposition. If $K: a \rightarrow d \mathcal{G}$ is a covariant functor, and $\chi: K \rightarrow \hat{K}$ a split representation, then

(1) $\chi: K^{R} \rightarrow \hat{K}^{R}$ is a split representation,

(2) if $\chi: K \rightarrow \hat{K}$ is weakly compatible with dimension, compatible with dimension, or normalized so is $\chi: K^{R} \rightarrow \hat{K}^{R}$,

(3) if $K$ is acyclic on models, so is $K^{R}$, and

(4) if $K$ is a singular functor, so is $K^{R}$.

The proofs of the preceding are straightforward, and we omit them. We now consider the same questions for the functor $K^{D}$. Here the situation is much more complicated.

4.4. Definition. Let $K: Q \rightarrow d g$ be a covariant functor, and $\chi: K \rightarrow \hat{K}$ a split representation of $K$. Define $\chi: K^{D} \rightarrow \hat{K}^{D}$ as follows: Suppose $u \in S(E)$, and $k \in K_{u}(E)$. Let $k^{\prime}$ be defined by $k^{\prime}=K(S(\alpha(\pi u), 1(B), u ; \pi u, \pi u ; \beta(\pi u)$, $\pi) \sigma(\pi u)) \theta(u) \chi(E) k$, and define $\chi(\pi) k=i\left(\beta(\pi u)^{\pi}\right) k^{\prime}$.

4.5. Proposition. Under the conditions of the preceding definition $\chi$ : $K^{D} \rightarrow \hat{K}^{D}$ is a split representation.

Proof. The most difficult thing to establish is the naturality of $\chi$, i.e. to prove that $K^{D}(F) \chi(\pi)=\chi\left(\pi^{\prime}\right) K^{D}(F)$. To do this we use three diagrams, Figures $1,2,3$, and the notation of these diagrams. 
We have

$$
g \beta(\pi u) P=g \pi Q=\pi^{\prime} f Q=\pi^{\prime} Q^{\prime} S^{\prime},
$$

and

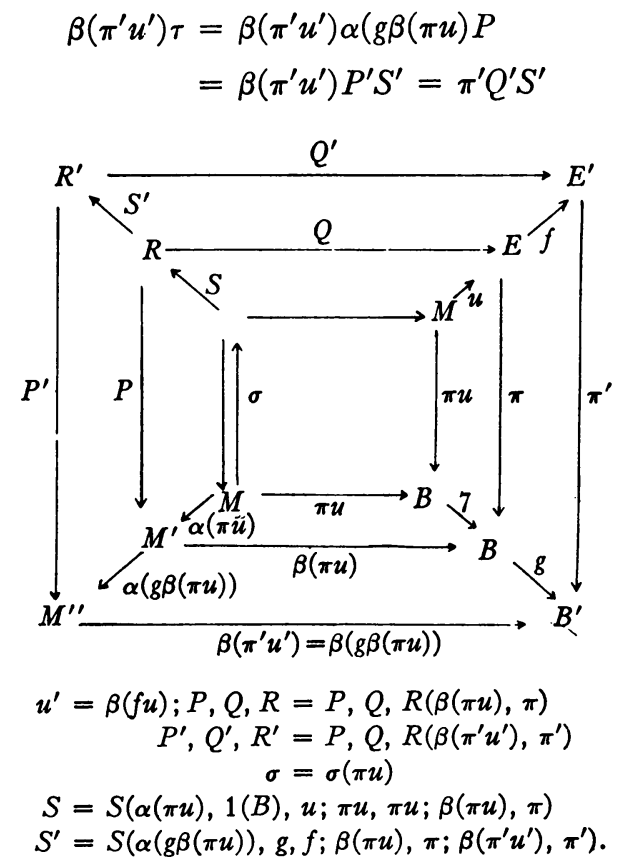

Fig. 1
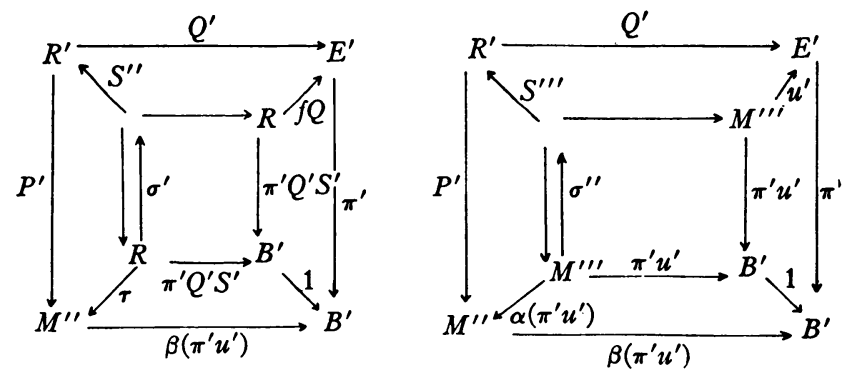

$$
\begin{aligned}
\tau & =\alpha(g \beta(\pi u)) P \\
\sigma^{\prime} & =\alpha\left(\pi^{\prime} Q^{\prime} S^{\prime}\right) \\
S^{\prime \prime} & =S\left(\tau, 1\left(B^{\prime}\right), f Q ; \pi^{\prime} Q^{\prime} S^{\prime}, \pi^{\prime} Q^{\prime} S^{\prime} ; \beta\left(\pi^{\prime} u^{\prime}\right), \pi^{\prime}\right) \\
\sigma^{\prime \prime} & =\sigma\left(\pi^{\prime} u^{\prime}\right) \\
S^{\prime \prime \prime} & =S\left(\alpha\left(\pi^{\prime} u^{\prime}\right), 1\left(B^{\prime}\right), u^{\prime} ; \pi^{\prime} u^{\prime}, \pi^{\prime} u^{\prime} ; \beta\left(\pi^{\prime} u^{\prime}\right), \pi^{\prime}\right)
\end{aligned}
$$

Figs. 2 and 3

(cf. Figures 1 and 2).

Now using the notation of 4.4 , we have 


$$
\begin{aligned}
\hat{K}^{D}(F) \chi k & =\hat{K}^{D}(F) i\left(v^{\pi}\right) k^{\prime} \\
& =i\left(\beta\left(F v^{\pi}\right)\right) \hat{K}^{D}\left(\beta\left(F v^{\pi}\right)\right) k^{\prime} \\
& =i\left(\beta\left(F v^{\pi}\right)\right) k_{1} .
\end{aligned}
$$

Referring to 3.8 ,

$$
\begin{aligned}
k_{1} & =K\left(S^{\prime \prime} \sigma^{\prime} S\right) \theta(u) \chi(E) k, \\
\chi K^{D}(F) k^{\prime} & =\chi(E) K(f) k \\
& =\chi \Psi(\beta(f u)) K(f) k \\
& =i\left(\beta\left(\pi^{\prime} u^{\prime}\right) \pi^{\prime}\right) k_{2}
\end{aligned}
$$

where

$$
k_{2}=K\left(S^{\prime \prime \prime} \sigma^{\prime \prime}\right) \theta\left(u^{\prime}\right) \chi\left(E^{\prime}\right) K(f) k .
$$

Now

$$
\begin{aligned}
\beta\left(F v^{\pi}\right) & =\left(Q\left(\beta(g \beta(\pi u)), \pi^{\prime}\right), \beta(g \beta(\pi u)) ; P^{\prime}, \pi^{\prime}\right) \\
& =\left(Q\left(\beta(g \pi u), \pi^{\prime}\right), \beta(g \pi u) ; P^{\prime}, \pi^{\prime}\right) \\
& =\left(Q\left(\beta\left(\pi^{\prime} f u\right), \pi^{\prime}\right), \beta\left(\pi^{\prime} f u\right) ; P^{\prime}, \pi^{\prime}\right) \\
& =\left(Q\left(\beta\left(\pi^{\prime} u^{\prime}\right), \pi^{\prime}\right), \beta\left(\pi^{\prime} u^{\prime}\right) ; P^{\prime}, \pi^{\prime}\right) \\
& =\beta\left(\pi^{\prime} u^{\prime}\right) \pi^{\prime},
\end{aligned}
$$

and hence by (1) and (3) above our result will follow if we show $k_{1}=k_{2}$.

$$
\begin{aligned}
& k_{2}=K\left(S^{\prime \prime \prime} \sigma^{\prime \prime}\right) \theta\left(u^{\prime}\right) \chi\left(E^{\prime}\right) K(f) k \\
& =K\left(S^{\prime \prime \prime} \sigma^{\prime \prime}\right) \theta\left(u^{\prime}\right) \widehat{K}(f) \tau(u) \chi(E) k \\
& =K\left(S^{\prime \prime \prime} \sigma^{\prime \prime}\right) \theta\left(u^{\prime}\right) \tau\left(u^{\prime}\right) K(\alpha(f u) \theta(u) \chi(E) k \\
& =K\left(S^{\prime \prime \prime} \sigma^{\prime \prime} \alpha(f u)\right) \theta(u) \chi(E) k \text {, }
\end{aligned}
$$

and comparing with (2), it remains to show that

$$
S^{\prime \prime} \sigma^{\prime} S \sigma=S^{\prime \prime \prime} \sigma^{\prime \prime} \alpha(f u) .
$$

Now,

$$
\begin{aligned}
Q^{\prime} S^{\prime \prime \prime} \sigma^{\prime \prime} \alpha(f u) & =u^{\prime} \alpha(f u)=\beta(f u) \alpha(f u)=f u, \\
Q^{\prime} S^{\prime \prime \prime} \sigma^{\prime} S & =f Q S \sigma=f u, \\
P^{\prime} S^{\prime \prime \prime} \sigma^{\prime \prime} \alpha(f u) & =\alpha\left(\pi^{\prime} u^{\prime}\right) \alpha(f u)=\alpha\left(\pi^{\prime} f u\right), \\
P^{\prime} S^{\prime \prime} \sigma^{\prime} S & =\alpha(g \beta(\pi u))=P S \sigma \\
& =\alpha(g \beta(\pi u)) \alpha(\pi u) \\
& =\alpha(g \pi u)=\alpha\left(\pi^{\prime} f u\right) .
\end{aligned}
$$


Therefore $S^{\prime \prime} \sigma^{\prime \prime} S \sigma=S^{\prime \prime \prime} \sigma^{\prime \prime} \alpha(f u)$ by condition (3) of 3.3. This completes the verification of naturality. The remainder of the proof that $\chi: K^{D} \rightarrow \hat{K}^{D}$ is a split representation is not difficult, and will be left to the reader.

The proposition just proved is the second key proposition in our calculation of $E^{2}$ of the spectral sequence of a fibre space, for it is this proposition which leads to the representability of $E^{1}$.

4.6. Definition. If $K: Q \rightarrow d \mathcal{G}$ is a covariant functor, a split representation $\chi: K \rightarrow \hat{K}$ is strongly compatible with dimension if it is compatible with dimension and if $\Psi(v) d \Psi(u) \neq 0$ implies $\gamma(\beta(\pi v))<\gamma(\beta(\pi u))$ or $\beta(\pi v)=\beta(\pi u)$ for $u, v \in S(A)$ and any map $\pi: A \rightarrow B$.

4.7. THEOREM. If $K: Q \rightarrow d g$ is a covariant functor, and $\chi: K \rightarrow \hat{K}$ is a split representation which is strongly compatible with dimension, then $K^{R}, K^{D}$ : $\mathrm{D} Q \rightarrow d \mathcal{G}$ have split representations which are compatible with dimension.

This theorem is essentially just a summary of what we have already proved. However, we now have by $\S 2$, a spectral sequence $E^{r} K^{D}: D Q \rightarrow d \mathcal{D}$, and we know a little bit about this spectral sequence.

4.8. Theorem. With the same hypotheses as in 4.7 , the functor $E_{*, a}^{1} K^{D}$ : $D Q \rightarrow d \subseteq$ has a normalized split representation.

5. The calculation of $E^{2}$. We now calculate $E^{2}$ in an abstract situation which parallels the calculation for fibre spaces, and as we shall see later actually gives us the theorem of Leray and Serre that $E^{2}(f)=H(B ; H(F))$ where $f: E \rightarrow B$ is a fibre map with fibre $F$. In this section we continue with the hypotheses that $(\alpha, \mathfrak{T}, \alpha, \beta, \gamma)$ and $\Sigma$ satisfy the conditions of $\S 4$.

5.1. Definitions. A sub-category $\mathcal{F}$ of $D Q$ is closed relative to $\Sigma$ if it satisfies the following conditions (cf. II.3.2, II.3.3):

(1) If $(f, \pi)$ is an object of $\widetilde{a}$ and $\pi$ an object of $\mathcal{F}$, then $\Sigma(f, \pi)$ is a map of $\mathcal{F}$.

(2) If $J:(f, \pi) \rightarrow\left(f^{\prime}, \pi^{\prime}\right)$ is a map of $\widetilde{a}$ and $\pi, \pi^{\prime}$ are objects of $F$, then $\Sigma(J)$ is a map of $D \mathcal{F}$.

When $\mathscr{F} \subset D Q$ is closed, define $\mathscr{F} \mathscr{T}$ to be $\mathscr{F} \cap D \mathscr{M}$, and let $\alpha, \beta, \gamma$ denote the restrictions of the degeneracies on $D Q$, and the dimension function.

In the preceding one thinks of $Q$, as the category of topological spaces and continuous maps, and of $\mathcal{F}$ as the subcategory of $D Q Q$ consisting of fibre spaces and fibre-preserving maps. The fact that $\mathcal{F}$ is closed is just the assertion that the induced space of a fibre space is again a fibre space, and that certain maps involved with the induced space of a fibre space are fibre-preserving maps. This situation will be considered in detail later.

5.2. Proposition. If $\mathfrak{F} \subset D Q$ is closed, then (F, $F \mathfrak{T}, \alpha, \beta, \gamma)$ is a category with models, degeneracies, and dimension; further the system of degeneracies $\alpha, \beta$ is weakly compatible with dimension.

5.3. Proposition. Let $F \subset D Q$ be closed, and let $L: D Q \rightarrow d G$ be a covariant 
functor. If $\chi: L \rightarrow \hat{L}$ is a representation which is split, compatible with dimension, or normalized, then $\chi|\mathcal{F}: L| \mathcal{F} \rightarrow \hat{L} \mid \mathcal{F}$ is a representation which is split, compatible with dimension, or normalized.

5.4. ThEOREM. Let $\mathcal{F} \subset D Q$ be closed, $L: \mathcal{F} \rightarrow d \mathcal{G}$ be a covariant functor, and $\chi: L \rightarrow \hat{L}$ a split representation which is compatible with dimension. Suppose $E_{*, q}^{1} L: \mathcal{F} \rightarrow d \mathcal{S}$ is augmentable and acyclic on models, and let $G(q)=G\left(E_{*, q}^{1} L\right)$ $=E_{0, q}^{2} L \mid \mathfrak{T}$. Finally, let $K: \mathcal{F} \rightarrow d \mathcal{G}$ be a singular functor, then the functors $E_{p, q}^{2} L$ and $H_{p}(K * G(q))$ are naturally equivalent.

Proof. By $2.5, E^{1} L$ has a split representation. The result now follows from I.2.16.

Using the preceding proposition and 4.3 a singular functor on $\mathcal{F}$ could be obtained by taking a singular functor $C$ on $Q$, and then using $C^{R} \mid \mathcal{F}$.

Having proved this proposition we now only have to verify its hypotheses in the case of fibre spaces and fibre preserving maps to calculate $E^{2}$ for a fibre space.

\section{The Singular theories on topological SPACES}

Throughout this chapter we will deal wth the category of topological spaces and continuous maps. When necessary this category will be denoted by $a$. Though we have seen how to define models, degeneracies, and dimension in this category in earlier examples (cf. I.1.2, I.1.7, I.1.13), we now want to do this in several ways.

1. The cubical singular theory. Recall that we have let $I^{0}$ denote a fixed point, and $I^{n}$ the unit $n$-cube. For $n>0, I^{n}$ is the set of $n$-tuples of real numbers $\left(t_{1}, \cdots, t_{n}\right)$ such that $0 \leq t_{i} \leq 1$ for $i=1, \cdots, n$.

1.1. Definitions. Let $u: I^{n} \rightarrow A$ be a singular $n$-cube. If $n>0$, define $\lambda_{i}^{\epsilon} u: I^{n-1} \rightarrow A$ by $\lambda_{i}^{\epsilon} u\left(t_{1}, \cdots, t_{n-1}\right)=u\left(t_{1}, \cdots, t_{i-1}, \epsilon, t_{i}, \cdots, t_{n}\right)$ where $\epsilon=0$ or 1 , and $i=1, \cdots, n$. Similarly for any $n$ define $\sigma_{i} u: I^{n+1} \rightarrow A$ by $\sigma_{i} u\left(t_{1}, \cdots\right.$, $\left.t_{n+1}\right)=u\left(t_{1}, \cdots, t_{i-1}, t_{i+1}, \cdots, t_{n+1}\right)$ for $i=1, \cdots, n+1$. The $\lambda_{i}^{\epsilon}$ are called "face operators" and the $\sigma_{i}$ "degeneracy operators."

1.2. Lemma. The face and degeneracy operators for singular cubes satisfy the following identities:

$$
\begin{array}{rlrl}
\lambda_{i}^{\epsilon} \lambda_{j}^{\epsilon^{\prime}} & =\lambda_{j}^{\epsilon^{\prime}} \lambda_{i+1}^{\epsilon} & & i \geq j, \\
\lambda_{i}^{\epsilon} \sigma_{j} & =\sigma_{j-1} \lambda_{i}^{\epsilon} & & i<j, \\
\lambda_{i}^{\epsilon} \sigma_{i} & =1, & & \\
\lambda_{i}^{\epsilon} \sigma_{j} & =\sigma_{j} \lambda_{i-1}^{\epsilon} & i>j,
\end{array}
$$

and

$$
\sigma_{i} \sigma_{j}=\sigma_{j+1} \sigma_{i}
$$$$
i \leq j \text {. }
$$

1.3. Definitions. Let $u: I^{n} \rightarrow A$ be a singular $n$-cube. 
(I) If $u \neq \sigma_{n-1} v$, define $\alpha(u)=1\left(I^{n}\right)$ and $\beta(u)=u$. Otherwise suppose $p+q$ $=n, u=\sigma_{n-1} \cdots \sigma_{p} v$ where $v$ is a singular $p$-cube such that $v \neq \sigma_{p-1} w$ for any singular cube $w$. Define

$$
\alpha(u)=\sigma_{n-1} \cdots \sigma_{p} 1\left(I^{p}\right),
$$

and

$$
\beta(u)=v .
$$

(II) If $u \neq \sigma_{j} v$ for any $j$ and any $v$, define $\alpha(u)=1\left(I^{n}\right)$ and $\beta(u)=u$. Otherwise suppose $p+q=n, u=\sigma_{j_{q}} \cdots \sigma_{j_{1}} v$ where $j_{1}<\cdots<j_{q}$ and $v \neq \sigma_{j} w$ for any singular cube $w$ and any $j$. Define

$$
\alpha(u)=\sigma_{j_{q}} \cdots \sigma_{j_{1}} 1\left(I^{p}\right),
$$

and

$$
\beta(u)=v .
$$

Note that in I above we have $\alpha(u)\left(t_{1}, \cdots, t_{n}\right)=\left(t_{1}, \cdots, t_{p}\right)$ and $\beta(u)\left(t_{1}, \cdots, t_{p}\right)=u\left(t_{1}, \cdots, t_{p}, 0, \cdots, 0\right)$ (cf. I.1.2).

1.4. Proposition. Let $\mathfrak{T}$ be the subcategory of a such that the objects of $\mathfrak{T}$ are cubes, and the maps are maps whose domain and range are a cube.

(1) If $\alpha, \beta:[\Re, a] \rightarrow[\Re, Q]$ are defined by I above, then $\alpha, \beta$ is a system of degeneracies on $Q$. (This system of degeneracies is known as the AFT SYSTEM OF DEGENERACIES).

(2) If $\alpha, \beta:[\mathfrak{T}, Q] \rightarrow[\mathfrak{T}, Q]$ are defined by II above, then $\alpha, \beta$ is a system of degeneracies on $Q$. (This system of degeneracies is known as the SYMMETRIC SYSTEM OF DEGENERACIES.)

1.5. Definitions. Recall that we have defined $Q_{n}(A)$ to be the free module generated by the singular $n$-cubes of $A$, for any topological space (cf. I.1.7) and $D_{n}(A)$ to be the submodule of $Q_{n}(A)$ generated by the degenerate $n$-cubes of $A$ with respect to the aft system of degeneracies. Let $D_{n}^{\prime}(A)$ be the submodule of $Q_{n}(A)$ generated by the degenerate $n$-cubes of $A$ with respect to the symmetric system of degeneracies. Let $C_{n}(A)=Q_{n}(A) / \Gamma{ }_{.}(A)$, $C_{n}^{\prime}(A)=Q_{n}(A) / D_{n}^{\prime}(A), C(A)=\sum C_{n}(A)$, and $C^{\prime}(A)=\sum C_{n}^{\prime}(A)$.

Define $d: Q(A) \rightarrow Q(A)$ by $d u=\sum_{i=1}^{n}(-1)^{i}\left(\lambda_{i}^{0} u-\lambda_{i}^{1} u\right)$ when $u$ is a singular $n$-cube of $A$.

When using the aft system of degeneracies, define $\chi: C \rightarrow \hat{C}$ by: $\chi u$ is the element of $C_{n}\left(I^{n}, u\right)$ corresponding to $1: I^{n} \rightarrow I^{n}$ for every nondegenerate cube $u$. Similarly when using the symmetric system of degeneracies define $\chi: C^{\prime} \rightarrow \hat{C}^{\prime}$ in the same manner.

Now proceed as in I.1.13. Define $D: I^{n} \times I^{n}$ by $D\left(t_{1}, \cdots, t_{n}, t\right)$ $=\left(t t_{1}, \cdots, t_{n}\right)$ and $U: Q\left(I^{n}\right) \rightarrow Q\left(I^{n}\right)$ by $U v\left(t_{1}, \cdots, t_{q+1}\right)=D\left(v\left(t_{2}, \cdots, t_{q+1}\right)\right.$, $\left.t_{1}\right)$ for $v: I^{a} \rightarrow I^{n}$. If $v$ is degenerate $U v$ is degenerate regardless of whether we are using aft or symmetric degeneracies. 
Finally if $u$ is a singular $n$-cube define $\gamma(u)=n, \gamma$ is a dimension function.

1.6. TheOREM. Both the aft and the symmetric system of degeneracies on $A$ are compatible with dimension. The singular functors $C$ and $C^{\prime}$ are acyclic on models with respect to either system of degeneracies. The functor $C$ has a split representation which is strongly compatible with dimension when using the aft system of degeneracies, and the functor $C^{\prime}$ has a split representation which is strongly compatible with dimension when using the symmetric system of degeneracies.

\subsection{Corollary. $H C$ and $H C^{\prime}$ are naturally equivalent.}

In fact we have $D_{n}^{\prime} \supset D_{n}$ and therefore there is a natural map of $C$ into $C^{\prime}$ and this map induces a natural equivalence of $H C$ and $H C^{\prime}$ [4].

\section{The simplicial singular theory.}

2.1. Definitions. Let $\Delta^{0}=I^{0}$, and let $\Delta^{n}$ be the set of $(n+1)$-tuples of real numbers $\left(r_{0}, \cdots, r_{n}\right)$ such that $\sum_{i=0}^{n} r_{i}=1$, and $r_{i} \geq 0$. The space $\Delta^{n}$ is the standard $n$-simplex and a map $u: \Delta^{n} \rightarrow A$ is called a singular $n$-simplex of $A$.

Let $\mathfrak{T}$ be the subcategory of $a$ such that the objects of $\mathfrak{T}$ are the standard simplexes, and the maps are just the maps in $Q$ whose domain and range are standard simplexes. Define a dimension function $\gamma$ on $A$ by defining $\gamma(u)=n$ where $u: \Delta^{n} \rightarrow A$.

Let $u: \Delta^{n} \rightarrow A$ be a singular $n$-simplex. If $n>0$, define $\partial_{i} u: \Delta^{n-1} \rightarrow A$ by $\partial_{i} u\left(r_{0}, \cdots, r_{n-1}\right)=u\left(r_{0}, \cdots, r_{i-1}, 0, r_{i}, \cdots, r_{n-1}\right)$ for $i=0, \cdots, n$. For any $n$, define $s_{i} u: \Delta^{n+1} \rightarrow A$ by $s_{i} u\left(r_{0}, \cdots, r_{n+1}\right)=u\left(r_{0}, \cdots, r_{i-1}, r_{i}+r_{i+1}\right.$, $\left.r_{i+2}, \cdots, r_{n+1}\right)$. The $\partial_{i}$ are called "face operators" and the $s_{i}$ "degeneracy operators."

2.2. Lemma. The face and degeneracy operators for singular cubes satisfy the following identities:

and

$$
\begin{array}{rl}
\partial_{i} \partial_{j}=\partial_{j-1} \partial_{i} & i<j, \\
\partial_{i} s_{j}=s_{j-1} \partial_{i} & i<j, \\
\partial_{j} s_{j}=\partial_{j+1} s_{j}=1, & i>j+1, \\
\partial_{i} s_{j}=s_{j} \partial_{i-1} &
\end{array}
$$

$$
s_{i} s_{j}=s_{j+1} s_{i}
$$

2.3. Definitions. Let $u: \Delta^{n} \rightarrow A$ be a singular $n$-simplex. If $u \neq s_{j} v$ for any $j$ and any $v$, define $\alpha(u)=1\left(\Delta^{n}\right)$ and $\beta(u)=u$. Otherwise suppose $p+q=n$, $u=s_{j_{q}} \cdots s_{j_{1}} v$ where $j_{1}<\cdots<j_{q}$ and $v \neq s_{j} w$ for any singular simplex $w$ and any $j$. Define

$$
\alpha(u)=s_{j q} \cdots s_{j_{1}} 1\left(\Delta^{p}\right)
$$

and 


$$
\beta(u)=v .
$$

2.4. Lemma. If $\alpha, \beta:[\mathfrak{T}, a] \rightarrow[\mathfrak{T}, a]$ are defined as in the preceding definition, then $\alpha, \beta$ is a system of degeneracies on $a$ which is compatible with dimension.

Actually the system of degeneracies we have defined could be called the symmetric system of degeneracies for simplicial singular theory, and an aft system of degeneracies could also be defined, just as for cubical theory. Either of these systems of degeneracies works well as far as ordinary homology theory is concerned, or as far as the spectral sequences of fibre maps are concerned.

2.5. Definitions. For any space $A$, let $Q_{n}^{\prime \prime}(A)$ be the free module generated by the singular $n$-simplexes of $A$, and $Q^{\prime \prime}(A)=\sum Q_{n}^{\prime \prime}(A)$. Let $D_{n}^{\prime \prime}(A)$ be the submodule of $Q_{n}^{\prime \prime}(A)$ generated by the degenerate $n$-simplexes, let $C_{n}^{\prime \prime}(A)=Q_{n}^{\prime \prime}(A) / D_{n}^{\prime \prime}(A)$, and $C^{\prime \prime}(A)=\sum C_{n}^{\prime \prime}(A)$. Define $d: Q^{\prime \prime}(A) \rightarrow Q^{\prime \prime}(A)$ by $d u=\sum_{t=0}^{n}(-1)^{i} \partial_{i} u$ for $u$ a singular $n$-simplex, and noting that $d\left(D_{n}^{\prime \prime}(A)\right)$ $\subset D_{n-1}(A)$ also denote by $d$ the induced differential operator on $C^{\prime \prime}(A)$. Define $\chi: C^{\prime \prime} \rightarrow \hat{C}^{\prime \prime}$ by $\chi u$ is the element of $C_{n}^{\prime \prime}$. $\left(\Delta^{n}, u\right)$ corresponding to $1: \Delta^{n} \rightarrow \Delta^{n}$ for $u$ a nondegenerate $n$-simplex.

Next define $D: \Delta^{n} \times I \rightarrow \Delta^{n}$ by $D\left(r_{0}, \cdots, r_{n}, t\right)=\left(1-t+t r_{0}, t r_{1}, \cdots, t r_{n}\right)$ and define $U: Q^{\prime \prime}\left(\Delta^{n}\right) \rightarrow Q^{\prime \prime}\left(\Delta^{n}\right)$ by

$$
U v\left(r_{0}, \cdots, r_{q+1}\right)=D\left(v\left(\frac{r_{1}}{1-r_{0}}, \cdots, \frac{r_{q+1}}{1-r_{0}}\right), 1-r_{0}\right)
$$

for $v: \Delta^{q} \rightarrow \Delta^{n}$. If $v$ is degenerate $U v$ is degenerate, and we also denote by $U: C^{\prime \prime}\left(\Delta^{n}\right) \rightarrow C^{\prime \prime}\left(\Delta^{n}\right)$ the induced homomorphism of degree +1 .

2.6. THEOREM. The singular functor $C^{\prime \prime}$ is acyclic on models and has a split representation which is strongly compatible with dimension.

To prove this theorem it suffices to note that if $u: \Delta^{n} \rightarrow \Delta^{m}$ and $\alpha(u)=u$, then $D\left(u\left(r_{0}, \cdots, r_{n}\right) t\right)=u\left(D\left(r_{0}, \cdots, r_{n}\right), t\right)$.

3. General homotopies in the singular theories and the prismatic theory. At this stage we are missing two important things: First we have not compared cubical singular theory with simplicial singular theory, and second we have not yet considered the effect on these theories of general homotopies between maps. To remedy the first situation we change our definition of the model sub-category of $a$, by letting $\mathfrak{T}$ be the category such that an object is either a standard cube or a standard simplex, with maps being all maps in $a$ whose domain and range are in $\mathfrak{T}$. Further we define a system of degeneracies $\alpha, \beta$ on $Q$. Suppose $u: M \rightarrow A$, then $\alpha(u)$ and $\beta(u)$ are defined by 1.3, II if $M$ is a cube, and by 2.3 if $M$ is a simplex. Finally we define a dimension function $\gamma$ by taking obvious extension of the simplicial and cubical dimension functions. 
Instead of the preceding we could have used aft degeneracies on the cubical half of the model sub-category. This would be convenient if we wanted to compare the functors $C$ and $C^{\prime \prime}$ directly. However, we choose to compare $C^{\prime}$ and $C^{\prime \prime}$ since it seems to us that the symmetric system of degeneracies is actually more convenient than the aft.

3.1. Theorem. On the category with models, degeneracies, and dimension defined above, the singular functors $C^{\prime}$ and $C^{\prime \prime}$ have split representations which are strongly compatible with dimension.

3.2. THEOREM. If $A$ is any topological space, and $G$ is a local coefficient system on $A$ (in the classical sense), then the cubical singular homology of $A$ with coefficients in $G$ is isomorphic with the simplicial singular homology of $A$ with coefficients in $G$.

The preceding, a slight strengthening of a theorem of Eilenberg-MacLane which states the same result for ordinary coefficients [4], follows from 3.1, and the work of I.3. In this theorem it makes no difference whether by the cubical singular homology we mean that obtained by using the chains normalized by dividing by the degenerate chains with respect to either the aft or the symmetric degeneracies, and by simplicial singular homology one can mean that obtained by dividing by the degeneracies of 2.3 , the aft degeneracies, or not dividing by degeneracies at all. To make this completely formal, it would be necessary to write a few more details, but this has not seemed worthwhile.

3.3. Definitions. The space $I^{r} \times \Delta^{n}$ is the standard $(r, n)$-prism. We consider $I^{r}$ as identified with $I^{r} \times \Delta^{0}$, and $\Delta^{n}$ as identified with $I^{0} \times \Delta^{n}$.

Let $P$ M denote the category whose objects are standard prisms, and whose maps are continuous maps between standard prisms. A map $u: I^{r} \times \Delta^{n} \rightarrow A$ is called a singular $(r, n)$-prism, and we denote by $Q_{r, n}(A)$ the free module generated by the singular $(r, n)$-prisms of $A$. Note that $Q_{0, n}(A)=Q_{n}^{\prime \prime}(A)$ and $Q_{r, 0}(A)=Q_{r}(A)$.

If $u: I^{r} \times \Delta^{n} \rightarrow A$, define

$$
\begin{aligned}
\lambda_{i}^{\epsilon} u\left(t_{1}, \cdots, t_{r-1}, r_{0}, \cdots, r_{n}\right) & =u\left(t_{1}, \cdots, t_{i-1}, \epsilon, t_{i}, \cdots, t_{r-1}, r_{0}, \cdots, r_{n}\right), \\
\sigma_{i} u\left(t_{1}, \cdots, t_{r+1}, r_{0}, \cdots, r_{n}\right) & =u\left(t_{1}, \cdots, t_{i-1}, t_{i+1}, \cdots, t_{r+1}, r_{0}, \cdots, r_{n}\right), \\
\partial_{j} u\left(t_{1}, \cdots, t_{r}, r_{0}, \cdots, r_{n-1}\right) & =u\left(t_{1}, \cdots, t_{r}, r_{0}, \cdots, r_{j-1}, 0, r_{j}, \cdots, r_{n-1}\right), \\
s_{i} u\left(t_{1}, \cdots, t_{r}, r_{0}, \cdots, r_{n+1}\right) & =u\left(t_{1}, \cdots, t_{r}, r_{0}, \cdots, r_{j-1}, r_{j}+r_{j+1}, r_{j+2}, \cdots, r_{n-1}\right) .
\end{aligned}
$$

The $\lambda_{i}^{e}$ 's and $\partial_{j}$ 's are the prismatic face operators, and the $\sigma_{i}$ 's and $s_{j}$ 's are the prismatic degeneracy operators.

3.4. Lemma. The prismatic face and degeneracy operators satisfy the identities of 1.2 and 2.2 and in addition the following commutation identities: 


$$
\lambda_{i}^{\epsilon} \partial_{j}=\partial_{j} \lambda_{i}^{\epsilon}, \quad \lambda_{i}^{\epsilon} s_{j}=s_{j} \lambda_{i}^{\epsilon}, \quad \sigma_{i} \partial_{j}=\partial_{j} \sigma_{i}
$$

and

$$
\sigma_{i} s_{j}=s_{j} \sigma_{i} \text {. }
$$

3.5. Definitions. Let $Q^{P}(A)=\sum_{r, n} Q_{r, n}(A)$, and define $d, d^{\prime}, d^{\prime \prime}$ : $Q^{P}(A) \rightarrow Q^{P}(A)$ by

$$
d^{\prime} u=\sum_{i=1}^{r}(-1)^{i}\left(\partial_{2}^{0} u-\partial_{i}^{1} u\right), \quad d^{\prime \prime} u=\sum_{j=1}^{n}(-1)^{i+r} \partial_{j} u,
$$

and

$$
d u=d^{\prime} u+d^{\prime \prime} u
$$

for $u$ a singular $(r, n)$ prism.

3.6. Lemma. Using the definitions of 3.5 we have

$$
\begin{aligned}
d^{\prime} d^{\prime} & =0, \\
d^{\prime \prime} d^{\prime \prime} & =0, \\
d^{\prime} d^{\prime \prime}+d^{\prime \prime} d^{\prime} & =0,
\end{aligned}
$$

and

$$
d d=0 .
$$

3.7. Definition. If $A$ is a topological space, define $S_{j}: Q_{r, n}(A) \rightarrow Q_{r-1, n+1}(A)$ as follows: Suppose $u: I^{r} \times \Delta^{n} \rightarrow A$ is a singular $(r, n)$ prism, then $S_{j} u\left(t_{1}, \cdots, t_{r}\right.$, $\left.r_{0}, \cdots, r_{n+1}\right)=u\left(1-\left(r_{0}+\cdots+r_{j}\right), t_{1}, \cdots, t_{r-1}, r_{0}, \cdots, r_{j-1}, r_{j}+r_{j+1}\right.$, $\left.r_{j+2}, \cdots, r_{n+1}\right)$ for $0 \leq j \leq n$.

3.8. Lemma. The following identities hold:

$$
\begin{array}{lr}
\partial_{i} S_{j}=S_{j-1} \partial_{i} & i<j, \\
\partial_{i} S_{j}=S_{j} \partial_{i-1} & i>j+1, \\
\partial_{j} S_{j}=\partial_{j} S_{j-1} & j>0, \\
\lambda_{i}^{\epsilon} S_{j}=S_{j} \lambda_{i+1}^{e}, & \\
\sigma_{i} S_{j}=S_{j} \sigma_{i+1}, & \\
\dot{S}_{j} \sigma_{1}=s_{j}, & \\
s_{i} S_{j}=S_{j+1} S_{i} & i \leq j, \\
s_{i} S_{j}=S_{j} S_{i-1} & i>j, \\
\partial_{0} S_{0}=\lambda_{1}^{1}, &
\end{array}
$$

and 


$$
\partial_{n+1} S_{n}=\lambda_{1}^{0}
$$

on $(r, n)$ prisms.

3.9. Definitions. Define

$$
\rho_{r}: Q_{r, n} \rightarrow Q_{r-1, n+1}
$$

by

$$
\rho_{r}=\sum_{j=0}^{n}(-1)^{j+r} S_{j} \quad r \geq 0 .
$$

Next define

$$
\rho: Q^{P} \rightarrow Q^{\prime \prime}
$$

by

$$
\rho \mid Q_{r, n}=\rho_{0} \cdots \rho_{r} .
$$

3.10. Lemma. We have the following identities:

$$
\begin{aligned}
d^{\prime} \rho_{r} & =\rho_{r-1}\left(d^{\prime}+\lambda_{1}^{0}-\lambda_{1}^{1}\right), \\
d^{\prime \prime} \rho_{r} & =\rho_{r} d^{\prime \prime}+\lambda_{1}^{1}-\lambda_{1}^{0},
\end{aligned}
$$

and

$$
d \rho=\rho d .
$$

Parts (1) and (2) of the lemma follow by a simple direct calculation using the identities of 3.8. Part (3) is proved by induction on $r$ using parts (1) and (2).

Suppose $u$ is a singular $(r, n)$ prism and $r>0$. Then

$$
\begin{aligned}
d \rho u & =d \rho_{0} \cdots \rho_{r-1} \rho_{r} u \\
& =d \rho \rho_{r} u \\
& =\rho d \rho_{r} u \text { by inductive hypothesis, } \\
& =\rho\left(d^{\prime}+d^{\prime \prime}\right) \rho_{r} u \\
& =\rho\left(\rho_{r-1}\left(d^{\prime}+\lambda_{1}^{0}-\lambda_{1}^{1}\right)+\rho_{r} d^{\prime \prime}+\lambda_{1}^{1}-\lambda_{1}^{0}\right) u \\
& =\rho\left(d^{\prime}+d^{\prime \prime}\right) u \\
& =\rho d u .
\end{aligned}
$$

Now using the preceding lemma we have a natural transformation of functors $\rho: Q^{P} \rightarrow Q^{\prime \prime}$. Actually this transformation is a retraction, it leaves $Q^{\prime \prime}$ fixed.

3.11. Proposition. If $F: I \times A \rightarrow B$ is a map, $f_{t}: A \rightarrow B$ is defined by $f_{t}(u)=F(t, a)$, and $K: Q \rightarrow d \mathcal{G}$ denotes one of the singular functors $C, C^{\prime}$, or $C^{\prime \prime}$, 
then there is a homomorphism of degree $+1, U: K(A) \rightarrow K(B)$ such that $d U+U d$ $=K\left(f_{0}\right)-K\left(f_{1}\right)$.

Proof. Suppose first that $K$ is one of the singular cubical functors, and define $U v\left(t_{1}, \cdots, t_{n+1}\right)=F\left(t_{1}, v\left(t_{2}, \cdots, t_{n+1}\right)\right)$ for $v$ a singular $n$-cube of $A$.

Suppose now $K$ is the simplicial cubical functor. Define $U^{\prime}: Q^{P} \rightarrow Q^{P}$ as follows: Let $v$ be a singular $(r, n)$ prism of $A$, let $U^{\prime} v\left(t_{1}, \cdots, t_{r+1}, r_{0}, \cdots, r_{n}\right)$ $=F\left(t_{1}, v\left(t_{r}, \cdots, t_{r+1}, r_{0}, \cdots, r_{n}\right)\right)$. Now let $v$ be a singular $n$-simplex of $A$, and let $i: Q^{\prime \prime}(A) \rightarrow Q^{P}(A)$ be the natural inclusion map, define $U v=\rho U^{\prime} i(v)$.

The preceding is one of the main reasons for introducing the functor $Q^{P}$. Actually we could make a category with models, degeneracies and dimension $(A, P \mathscr{T} \alpha, \beta, \gamma)$ by using the combined simplicial and cubical degeneracies. On this category we could define a singular functor $C^{P}$ which is the quotient of $Q^{P}$ by the submodule generated by the degenerate prisms. However, there seems to be no particular interest in this functor except as a means of relating the cubical and simplicial singular functors.

\section{FIBRE SPACES}

Throughout this chapter, $a$ is the category of topological spaces and continuous maps.

1. The calculation of $E^{2}$ for a fibre space.

1.1. Proposition. There exists an induced structure $\Sigma=\Sigma(P, Q, R, S)$ on $a$.

Proof. For any $A \stackrel{\rho}{\rightarrow} B \underset{\leftarrow}{\leftarrow} E$ in $A$, we define $R(f, \pi)=\{(a, e) \mid(a, e) \in A \times E$, $f a=\pi e\}$ with the topology induced by that of $A \times E$ (cf. II.3.1). We then define $Q(f, \pi), P(f, \pi), \sigma(\pi)$ by

$$
\begin{aligned}
& Q(f, \pi)(a, e)=e, \\
& P(f, \pi)(a, e)=a,
\end{aligned}
$$

and

$$
\sigma(\pi) e=(e, e) .
$$

Finally we define $S\left(h, k, g ; f, \pi ; f^{\prime}, \pi^{\prime}\right)=S$ by

$$
S(a, e)=(h a, g e) \text {. }
$$

1.2. Definition. A map $\pi: E \rightarrow B$ will be called a fibre map if it is onto and satisfies the following condition:

Suppose $P$ is a finite convex polyhedron with boundary $\bar{P}$, and maps $F: I \times P \rightarrow B, f: I \times \bar{P} \cup\{0\} \times P \rightarrow E$ are given such that $\pi f=F \mid I \times P \cup\{0\} \times P$, then there is a map $\tilde{F}: I \times P \rightarrow E$ such that $\pi \tilde{F}=F$ and $\tilde{F} \mid I \times P \cup\{0\} \times P=f$.

As is well known [3, Proposition 1, p. 443] this is precisely equivalent to the definition of fibre space of Serre. 
1.3. Notation. We will denote by $\mathcal{F}$ the sub-category of $\mathscr{D} Q$ the objects of which are fibre maps, and the maps all maps of $D Q$ with fibre maps as domain and range.

\subsection{Lemma. The sub-category $\mathcal{F}$ of $D Q$ is closed relative to $\Sigma$ and $\bar{\Sigma}$.}

1.5. Theorem. Let $L: \mathcal{F} \rightarrow d \mathcal{G}$ be one of the functors $C^{D}\left|\mathcal{F}, C^{D}\right| \mathcal{F}$, or $C^{\prime \prime D} \mid \mathcal{F}$, let $K: \mathcal{F} \rightarrow d \mathcal{G}$ be the corresponding functor $C^{R}\left|\mathcal{F}, C^{\prime R}\right| \mathcal{F}$, or $C^{\prime \prime R} \mid \mathcal{F}$, and let $G(q)=E_{0,8}^{2} L \mid M$. Under these conditions the functors

$$
E_{p, q}^{2} L \text { and } H_{p}(K * G(q))
$$

are naturally equivalent.

To prove this theorem, it suffices by II.5.3 and II.5.4 to prove that $E_{*, q}^{1} L$ is augmentable and acyclic on models. This will be taken care of in $\S \S 3$ and 4.

The preceding theorem is the theorem which gives the structure of $E^{2}$ for the spectral sequence of a fibre space. In more classical language it says that if $\pi: E \rightarrow B$ is a fibre map with fibre $F$, then $E^{2}(\pi) \simeq H(B ; H(F))$, the coefficients on the right being local, and that this isomorphism is natural on the category $F$.

2. Fibres. If $\pi: E \rightarrow B$ is a fibre map, the counterimage of a point $b \in B$ is the fibre over $b$. For sufficiently smooth fibre spaces with connected base space $B$, the fibres over any two points have the same homotopy type, and in general their singular complexes have the same homotopy type when the base space $B$ is connected.

Before proceeding with the remainder of the proof of the last theorem, we wish to prove it for the special case where $B=b$ is a single point. Note that any map with image a single point is a fibre map.

2.1. Proposition. If $\pi: E \rightarrow b$ is a map where $b$ is a single point and $L$ is as in 1.5 , then

$$
E_{p, q}^{r} L(\pi)=0 \text { for } p>0 \text { and all } r
$$

and

$$
E_{0, q}^{r} L(\pi)=H_{q} L(\pi) .
$$

The proof of this proposition is immediate.

3. Geometric considerations. For this section we let $(Q, \mathfrak{T}, \alpha, \beta, \gamma)$ denote the category with models, degeneracies and dimension of III.3. In this section and the next we will give proofs assuming the covering homotopy theorem for arbitrary spaces. These proofs, by a slight modification, work for the singular cubical complex or the singular simplicial complex with definition of fibre space being used here. The details of carrying through the modifications are 
sometimes a little tedious, but no new ideas are involved.

Notation. Let $A, B$ be spaces. If $F: I \times A \rightarrow B$ is a map let $F_{t}: A \rightarrow B$ be defined by $F_{t}(a)=F(t, a)$.

3.1. Proposition. Let $\pi: E \rightarrow B, \pi^{\prime}: E^{\prime} \rightarrow B^{\prime}, F: I \times B^{\prime} \rightarrow B$, and $f: E^{\prime} \rightarrow E$ be maps. Suppose $\pi$ is a fibre map and that $F_{0} \pi^{\prime}=\pi f$, then there exists $\tilde{F}: I \times E^{\prime} \rightarrow E$, a map such that

$$
\tilde{F}_{0}=f \text {, }
$$

and

$$
\pi \widetilde{F}_{t}=F_{t} \pi^{\prime}
$$

This proposition is just a statement of the covering homotopy theorem or condition.

3.2. Proposition. Under the same conditions as the preceding proposition assume that $\tilde{F}$ and $\tilde{F}^{\prime}: I \times E^{\prime} \rightarrow E$ are maps such that

and

$$
\widetilde{F}_{0}=\widetilde{F}_{0}^{\prime}=f,
$$

$$
\pi \tilde{F}_{t}=\pi \widetilde{F}_{t}^{\prime}=F_{t} \pi^{\prime} .
$$

In this case there exists a map

$$
G: I \times I \times E^{\prime} \rightarrow E
$$

such that

$$
\begin{aligned}
G\left(0, t^{\prime}, e^{\prime}\right) & =F\left(t^{\prime}, e^{\prime}\right), \\
G\left(1, t^{\prime}, e^{\prime}\right) & =F^{\prime}\left(t^{\prime}, e^{\prime}\right), \\
G\left(t, 0, e^{\prime}\right) & =f,
\end{aligned}
$$

and

$$
\pi G\left(t, t^{\prime}, e^{\prime}\right)=F_{t^{\prime}} \pi^{\prime}\left(e^{\prime}\right) .
$$

This proposition again follows immediately from the definition of covering homotopy. It says that the particular choice of a covering homotopy $\tilde{F}$ of $F$ does not make a great deal of difference.

3.3. Proposition. Suppose the conditions of 3.1 are fulfilled, then there is a homomorphism of degree +1

$$
V: Q\left(E^{\prime}\right) \rightarrow Q(E)
$$

such that $V$ maps cubes into cubes, and

$$
Q(\pi) V=U Q\left(\pi^{\prime}\right),
$$

where $U$ is as in III.3.11 relative to $F$, 


$$
\begin{aligned}
\lambda_{1}^{0} V & =Q(f), \\
\lambda_{i+1} V & =V \lambda_{i},
\end{aligned}
$$

and

$$
\sigma_{i+1} V=V \sigma_{i} .
$$

Comments on the proof. If we assume the existence of $\widetilde{F}$ as in 3.1 and define $V u\left(t_{1}, \cdots, t_{n+1}\right)=\tilde{F}\left(t_{1}, u\left(t_{2}, \cdots, t_{n+1}\right)\right)$ then $V$ satisfies the required conditions. However, to prove the existence of $V$ we may work by induction on dimension. Then if $V$ is defined and satisfies the stated conditions in dimensions less than $n$ and $u$ is an $n$-cube, our conditions impose certain restrictions on $V u$. It can be shown without too much difficulty that there always exists a cube $V u$ when $\pi$ is a fibre map (1.2).

3.4. Proposition. Suppose $V_{0}, V_{1}: Q\left(E^{\prime}\right) \rightarrow Q(E)$ satisfy the conditions of 3.3 , then there exists

$$
W: Q\left(E^{\prime}\right) \rightarrow Q(E)
$$

a homomorphism of degree +2 which maps cubes into cubes and such that

$$
\begin{aligned}
Q(\pi) W & =\sigma_{2} Q(\pi) V_{0}=\sigma_{2} Q(\pi) V_{1}, \\
\lambda_{1}^{0} W & =\sigma_{1} Q(f), \\
\lambda_{2}^{0} W & =V_{0}, \lambda_{2}^{1} W=V_{1}, \\
\lambda_{i+2}^{e} W & =W \lambda_{i}^{*},
\end{aligned}
$$

and

$$
\sigma_{i+2} W=W \sigma_{i} .
$$

Comments on the proof. This proposition bears the same analogy to Proposition 3.2 that Proposition 3.3 does to Proposition 3.1. The fact that these propositions are satisfied for any fibre space means more or less that when one is working with singular cubical theory the covering homotopy theorem for finite complexes is all that is necessary. The general covering homotopy theorem is convenient for if $G$ exists as in 3.2 and one defines $W v\left(t_{1}, \cdots, t_{n+2}\right)$ $=G\left(t_{2}, t_{1}, v\left(t_{3}, \cdots, t_{n+1}\right)\right)$ then $W$ satisfies the required conditions. However, the existence of $W$ can be proved by induction on dimension just as the existence of $V$ in the preceding proposition.

3.5. Proposition. Suppose the conditions of 3.1 are fulfilled, then there is a homomorphism

$$
V: Q^{\prime \prime}(E) \rightarrow Q^{P}(E)
$$

such that $V u$ is $a(1, n)$ prism for $u$ an $n$-simplex, and such that 


$$
\begin{aligned}
Q(\pi) V u\left(t_{1}, r_{0}, \cdots, r_{n}\right) & =F\left(t_{1}, \pi^{\prime} u\left(r_{0}, \cdots, r_{n}\right)\right), \\
\lambda_{1}^{0} V & =f_{0}, \\
\partial_{i} V & =V \partial_{i},
\end{aligned}
$$

and

$$
s_{i} V=V s_{i} .
$$

3.6. Proposition. Suppose $V_{0}, V_{1}: Q^{\prime \prime}\left(E^{\prime}\right) \rightarrow Q^{P}(E)$ satisfy the conditions of 3.5 , then there exists

$$
W: Q^{\prime \prime}\left(E^{\prime}\right) \rightarrow Q^{P}(E)
$$

such that $W u$ is $a(2, n)$ prism for $u$ on $n$-simplex, and such that

$$
\begin{aligned}
Q(\pi) W & =\sigma_{2} Q(\pi) V_{0}=\sigma_{2} Q(\pi) V_{1}, \\
\lambda_{1}^{0} W & =\sigma_{1} Q(f), \\
\lambda_{2}^{0} W & =V_{0}, \lambda_{2}^{1} W=V_{1}, \\
\partial_{i} W & =W \partial_{i},
\end{aligned}
$$

and

$$
s_{i} W=W s_{i} .
$$

Using the homomorphism $\rho$ of III.3.9 Propositions 3.5 and 3.6 play the same role for the simplicial singular theory as do 3.3 and 3.4 for the cubical singular theory.

3.7. Proposition. The homomorphisms $V$ of Propositions 3.3 and 3.5 are of degree +1 with respect to the filtrations involved. Further in the notation of 1.5 , there is a homomorphism of degree +1

$$
-V^{1}: E^{1} L\left(\pi^{\prime}\right) \rightarrow E^{1} L(\pi)
$$

of degree +1 induced by $V$ such that

$$
E^{1} L([f])-E^{1} L\left(\left[\lambda_{1}^{1} V\right]\right)=d^{1} V^{1}+V^{1} d^{1},
$$

and this homomorphism is independent of the choice of $V$.

In the preceding proposition $[f]$ is the map

$$
\begin{aligned}
& E^{\prime} \stackrel{f}{\longrightarrow} E \\
& \downarrow_{B^{\prime}}^{\pi^{\prime}} \stackrel{F_{0}}{\longrightarrow} \stackrel{\downarrow^{\pi}}{\longrightarrow}
\end{aligned}
$$

in $D Q$, and $\lambda_{1}^{1} V: Q\left(E^{\prime}\right) \rightarrow Q(E)$ is a filtration preserving chain map which in- 
duces a map of filtered modules $L\left(\pi^{\prime}\right) \rightarrow L(\pi)$. The existence of the homomorphism $V^{1}$ follows at once from 3.3 or 3.5. Further it is implied by 3.4 or 3.6 that if we have another such homomorphism $U$, then $-U^{1}=-V^{1}: E^{1} L\left(\pi^{\prime}\right)$ $\rightarrow E^{1} L(\pi)$.

4. The acyclicity on models and augmentability of the functors $E_{*, q}^{\prime}$. In this section we will let $L$ be one of the functors $C^{D}\left|F, C^{\prime D}\right| F$, or $C^{\prime \prime D} \mid F$, and we will denote by $E^{r}$ the functor $E^{r} L$.

Now we want to show that as far as the functor $E^{2}$ is concerned it makes no difference if the base space $B$ of $\pi: E \rightarrow B$ is a point, or a contractible space.

4.1. Proposition. Let $\pi: E \rightarrow B$ be a fibre map, $b^{\prime} \in B$, and $E^{\prime}=\pi^{-1}\left(b^{\prime}\right)$. Let $i: b^{\prime} \rightarrow B, i: E^{\prime} \rightarrow E$ be the inclusion maps, and $\pi^{\prime}=\pi \mid E^{\prime}$. Let $[i]=\left(i, i ; \pi^{\prime}, \pi\right)$, and let $F: I \times B \rightarrow B$ be a map such that $F(0, b)=b, F(1, b)=b^{\prime}$, and $F\left(t, b^{\prime}\right)=b^{\prime}$ for all $b \in B$ and $t \in I$. In this case

(1) $E^{2}([i])$ is an isomorphism,

(2) there are homomorphisms $V^{1}: E^{1}(\pi) \rightarrow E^{1}(\pi)$ and $\eta: E_{0}^{2}(\pi) \rightarrow E_{0}^{1}(\pi)$ such that

(i) $V^{1}\left(E_{p, q}^{1}(\pi)\right) \subset E_{p+1, q}^{1}(\pi)$,

(ii) if $V_{p}^{1}=V^{1} \mid E_{p}^{1}(\pi)$, then

$$
d^{1} V_{p}^{1}+V_{p-1}^{1} d^{1}=1 \text { for } p>0,
$$

and

$$
d^{1} V_{0}^{1}=1-\eta \epsilon .
$$

Proof. Proof using 3.3 or 3.5 there is a chain homotopy which lies over the chain homotopy induced by $F$. Denote this chain homotopy by $V$. The existence of $V^{1}$ now follows from 3.7.

Let $[g]: L(\pi) \rightarrow L\left(\pi^{\prime}\right)$ be the map induced by $\lambda_{1}^{1} V$. We then have

$$
d^{\prime} V^{\prime}+V^{\prime} d^{\prime}=1-E^{\prime}([i]) E^{\prime}([g])
$$

and hence $E^{2}([i]) E^{2}([g])=1$.

Now since $b^{\prime}$ is stationary under $F, V\left(L\left(\pi^{\prime}\right)\right) \subset L\left(\pi^{\prime}\right)$. Using this we see that $[g][i]$ is homotopic to the identity by a homotopy of degree zero with respect to the filtration on $L\left(\pi^{\prime}\right)$. Consequently $E^{1}([g]) E^{1}([i])=1$ and the proof of (1) is complete.

We have seen earlier that $E_{p}^{1}\left(\pi^{\prime}\right)=0$ for $p>0$. Hence $E_{p}^{1}([g])=0$ for $p>0$, and $d^{1} V_{p}^{1}+V_{p-1}^{1} d^{1}=1$.

It remains to deal with the case $p=0$. Let $\bar{\eta}=E_{0}^{1}([i]) E_{0}^{1}([g])$. Then $d^{1} V^{1}$ $=1-\bar{\eta}$. Suppose $x \in E^{1}(\pi)$, then $d^{1} x=E_{0}^{1}([i]) d^{1} E_{1}^{1}([g]) x=0$. Hence $\bar{\eta}$ annihilates the image of $d^{1}$ and induces $\eta: E_{0}^{2}(\pi) \rightarrow E_{0}^{1}(\pi)$ such that $\bar{\eta}=\eta \epsilon$, and the proof is complete.

4.2. Proposition. The functor $E_{*, \ell}^{1}$ is acyclic on models. 
Proof. In view of Proposition 4.1, it remains only to show that $V^{1}$ and $\eta$ can be chosen to be natural on $\mathscr{F I T}^{\alpha}$. This, however, follows from the fact that there are natural contracting homotopies of the base spaces, there exist homotopies which lie over these and they induce unique homotopies on $E^{1}$ by the uniqueness part of 3.7 .

\subsection{Proposition. The functor $E_{*, q}^{1}$ is augmentable.}

Proof. We need only to show that $[f]=\left(f, g ; \pi, \pi^{\prime}\right)$ is a nondegenerate map of $\mathscr{F} \mathscr{T}$, then $E_{0}^{2}([f])$ is an isomorphism. However, all nondegenerate maps in $\mathscr{F} \mathbb{T}$ are of the form $\Sigma\left(v, \pi^{\prime}\right)$ where $\pi^{\prime}: E^{\prime} \rightarrow M^{\prime}$, and $M^{\prime}$ is contractible. The result now follows from 4.1 , and the fact that any such $f$ when confined to a single fibre is a homeomorphism.

Now we have completed the proof of Theorem 1.5. In other words we have that if $\pi: E \rightarrow B$ is a fibre space with fibre $F$, then $E^{2}(\pi) \simeq H(B ; H(F))$.

5. Comparison of the singular spectral sequences. In this section we will denote by $E^{r}$ the terms of the spectral sequence arising from $C^{D} \mid \mathcal{F}$, by ' $E^{r}$ those arising from $C^{\prime D} \mid \mathcal{F}$, and by " $E^{r}$ those arising from $C^{\prime \prime} \mid \mathcal{F}$. We already have proved in Theorem 1.5 that the functors $E^{r},{ }^{\prime} E^{2}$, and " $E^{2}$ are naturally equivalent. Therefore, it remains to compare the later terms in these spectral sequences.

Notation. Let $\xi: C^{D} \rightarrow C^{\prime D}$ be the natural map, and let $\rho: C^{D} \rightarrow C^{\prime \prime} D$ be the map induced by the inclusion $i: C^{\prime D} \rightarrow C^{P, D}$ and the map $\rho: C^{P, D} \rightarrow C^{\prime \prime D}$ (cf. III.3.9).

5.1. Proposition. The map $\rho: C^{\prime D} \rightarrow C^{\prime \prime D}$ is filtration preserving.

Using the identities III.3.8 it is clear that $\rho: Q^{\prime D} \rightarrow Q^{\prime \prime D}$ does not decrease the number of degeneracies, and the result follows.

Notation. Let $\xi^{r}: E^{r} \rightarrow{ }^{\prime} E^{r}$ be the map induced by $\xi$, and $\rho^{r}:{ }^{\prime} E^{r} \rightarrow^{\prime \prime} E^{r}$ be the map induced by $\rho$.

5.2. THEOREM. If $r \geq 2$, then

(1) $\xi^{r}: E^{r} \rightarrow^{\prime} E^{r}$ is a natural equivalence, and

(2) $\rho^{r}: E^{r} \rightarrow{ }^{\prime \prime} E^{r}$ is a natural equivalence.

Proof. Using I.1.4 we see that $\xi^{2}$ and $\rho^{2}$ are natural equivalences. Now since $\xi^{r} d^{r}=d^{r} \xi^{r}$ and $\rho^{r} d^{r}=d^{r} \rho^{r}$, the result follows at once.

\section{REFERENCES}

1. J. Leray, L'anneau spectral et l'anneau filtre d'homologie d'une application continue, J. Math. Pures Appl. IX vol. 29 (1950) pp. 1-139.

2. - L'homologie d'une espace fibré dont la fibre est connexe, Ibid. vol. 29 (1950) pp. 169-213.

3. J-P. Serre, Homologie singulière des espaces fibrés. Applications, Ann. of Math. vol. 54 (1951) pp. 425-505. 
4. S. Eilenberg and S. MacLane, Acyclic models, Amer. J. Math. vol. 65 (1953) pp. 189199.

5. S. Eilenberg and H. Cartan, Homological algebra, Princeton University Press, 1956.

6. S. Eilenberg and S. MacLane, General theory of natural equivalences, Trans. Amer. Math. Soc. vol. 58 (1945) pp. 231-294.

7. S. Eilenberg and N. Steenrod, Foundations of algebraic topology, Princeton University Press, 1952.

8. N. E. Steenrod, Homology with local coefficients, Ann. of Math. vol. 44 (1945) pp. 142144.

Birkbeck College,

LONDON, ENGLAND

PRINCETON UNIVERSITY,

Princeton, N. J. 